\title{
HETEROGENEIDAD DE LA BRECHA DE GÉNERO EN LA PARTICIPACIÓN LABORAL DE LAS MIGRACIONES INTRARREGIONALES EN AMÉRICA LATINA (2010)
}

\author{
Fernando Ariel Manzano
}

Universidad Nacional del Centro de la Provincia de Buenos Aires

\begin{abstract}
RESUMEN
Desde la mitad del siglo XX en América Latina la migración intrarregional lleva el protagonismo, presentándose en los últimos años un aumento de esta tendencia, exhibiendo un peso relativo cada vez mayor. En el contexto de desarrollo de la región se destaca un aumento de la diversificación de la demanda de trabajo, dejando atrás las rígidas estructuras de género, al mismo tiempo que se produce una feminización de viejas ocupaciones, con diferencias entre los países de la región. Dado el incremento de la demanda de mano de obra de las mujeres, y las posibilidades de trabajo fuera del país de origen que afecta la movilidad laboral según género. Las nuevas oportunidades de trabajo y la posibilidad de generar un ingreso que no existían en los lugares de origen. El objetivo es cuantificar la variabilidad en los niveles de participaciones femeninas de las inmigrantes intrarregionales, en términos de la brecha de género, considerando los países de destino posible dentro de la región. Los insumos cuantitativos utilizados surgen de los resultados de los operativos censales latinoamericanos de la ronda de 2010, sobre los cuales se han realizado procesos de consistencia de manera de garantizar su comparabilidad.
\end{abstract}

Palabras claves: Migración intrarregional; Tasa de actividad; Brecha de género; Mercado de trabajo; Índice de masculinidad; América Latina.

\section{THE BREACH OF GENRE IN THE LABOR PARTICIPATION OF THE MIGRATIONS INTRARREGIONALES OF LATIN AMERICA (2010)}

\section{ABSTRACT}

From half of the XXth century in Latin America the migration intrarregional takes the leading role, presenting in the last years an increase of this tendency to him, exhibiting an every time major relative weight. In the context of development of the region one emphasizes an increase of the diversification of the demand of work, leaving behind the rigid genre structures, at the same time that a feminización of old occupations takes place, with differences between the countries of the region. Given the increase of the demand of labor of the women, and the possibilities of work out of the fatherland that affects the labor mobility as genre. The new opportunities of work and the possibility of generating a revenue that they did not exist in the origin places. The target is to quantify the changeability in the levels of feminine shares of the immigrants intrarregionales, in terms of the breach of genre, considering the countries of possible destination inside the region. The used quantitative inputs arise from the results of the operative Latin-American pensions of the round of 2010, on which there have been realized processes of consistency of way of guaranteeing its comparability.

Key words: Migration intrarregional; Activity valuation; Genre breach; Labor market; Masculinity index; Latin America.

Universidad Nacional del Centro de la Provincia de Buenos Aires. E-mail:
fernandoarielmanzano@fch.unicen.edu.ar 


\section{INTRODUCCIÓN}

América Latina se encuentra conformada por veinte países ${ }^{2}$, tres de ellos pertenecen a la región de El Caribe -Cuba, Haití y la República Dominicana-. La delimitación de este recorte geográfico bastante impreciso, se debe a diversas necesidades, propósitos y contextos (GRIMOLDI, 2014), vinculados al control durante 3 o 4 siglos por parte de España y Portugal, y a la posterior superación de esta experiencia colonial (BOHOSLAVSKY, 2009).

Existe abundancia de trabajos sobre la problemática migratoria en América Latina. Desde la segunda mitad del siglo XX, la migración latinoamericana puede reducirse a tres patrones de determinación relevante, estos son: en la época colonial el protagonismo lo tuvieron la inmigración histórica de ultramar ${ }^{3}$ (VILLA et al., 2002)-mantiene cierta vigencia, pero en relación a la décadas previas la intensidad y sobre todo su peso relativo es cada vez menor-; la emigración extrarregional ${ }^{4}$-una tendencia al descenso de los flujos a los principales destinos, aunque no por el momento de los stocks-; y los intercambios intrarregionales (MARTÍNEZ, CANO et al., 2014), cuya particularidad consiste en ser más inestable en comparación con las viejas corrientes transatlánticas (MARTÍNEZ et al., 2005). Este último fenómeno ha tendido a incrementarse en los últimos años, demandando mayor relevancia su indagación.

En el marco de una ausencia de proyectos de planificación sociales y demográficas por parte de los estados (ARUJ, 2008), los aumentos de la población migratoria producen efectos negativos en el mercado de trabajo - como ser el incremento de la discriminación y la xenofobia sobre los trabajadores migrantes (STALKER, 1994), disminución de los salarios de los trabajadores nativos por la competencia con los migrantes, entre otros-, así como también en el sistema de salud, en los servicios públicos, y en todas las estructuras de los países emisores y receptores de la región.

La introducción del género ${ }^{5}$, impulsó la relevancia analítica de considerar las diferencias sociales, demográficas, económicas y culturales entre mujeres, analizando las mismas al interior de grupos sociales determinados y contextos específicos (CHANT et al., 2003).

Conforme se transitan los procesos de desarrollo los mercados aumentan su expansión y la demanda de trabajo se diversifica, debilitándose las estructuras tradicionales ${ }^{6}$ de género (BOSERUP, 1970). Las oportunidades de nuevos puestos de trabajo sumado a los avances tecnológicos que permiten a las mujeres dedicar menos tiempo a las tareas del hogar - así como también elevar los niveles educativos-, incrementan la competitividad femenina en el mercado de trabajo (GOLDIN, 2006). De este modo, a medida que ocupaciones tradicionalmente realizadas por varones se "femenizan", pasan a recibir una menor valorización social y económica $^{7}$ (DAEREN, 2001).

\footnotetext{
${ }^{2}$ Argentina, Bolivia, Brasil, Chile, Colombia, Costa Rica, Cuba, Ecuador, El Salvador, Guatemala, Haití, Honduras, México, Nicaragua, Panamá, Paraguay, Perú, República Dominicana, Uruguay y Venezuela.

${ }^{3}$ Desde fines de siglo XIX hasta mediados del XX, movilizó a 55 millones de europeos aproximadamente y actuó como válvula de escape, posibilitando la organización o reorganización de los estados europeos (ARUJ, 2008).

${ }^{4}$ Comienza en las últimas décadas del siglo XX y continúa hasta la actualidad. Se estima que en todo el mundo, más de 150 millones de personas no residen actualmente en su país de origen (ARUJ, 2008). En el año 2000, según CEPAL (2004), un total de 20 millones de latinoamericanos vivían en un país diferente al de su nacimiento.

${ }^{5}$ Se reconoce el género como una categoría relacional, dinámica y socialmente construida (SZASZ, 1999). A nivel macro, el género determina la división sexual del trabajo y las prácticas de instituciones sociales y económicas tales como la familia, los mercados de trabajo, la religión, la ley, entre otros, otorgándoles valores, prestigio, actividades y remuneraciones diferenciados por género. Esta perspectiva trata de esclarecer cómo la división sexual del trabajo y los patrones de socialización prevalecientes privilegian a los hombres en detrimento de las mujeres.

${ }^{6}$ Según Boserup se dejó atrás un sistema de producción agrícola donde la mujer era el soporte básico de la familia y gozaba de cierta autonomía, para avanzar a un sistema donde las mujeres pasaron a ser trabajadoras no remuneradas dentro de una estructura familiar dominada por el jefe del hogar propietario o responsable de la tierra (BOSERUP, 1970).

${ }^{7}$ La división sexual del trabajo influye en la reproducción y la producción, a partir de dos principios: el de la separación, según el cual existen trabajos de hombres y trabajos de mujeres; y el de la jerarquización, el cual asigna mayor valor a los trabajos de hombres que a los de las mujeres (KERGOAT, 2003:847). Desde
} 
Se presenta como regular los niveles de participación femenina durante las etapas del desarrollo económico mediante una curva en forma de "U", que describiría altas tasas de actividad femenina en los estadios tempranos y tardíos del desarrollo y relativamente bajas en los períodos intermedios ${ }^{8}$ (GOLDIN, 1994; GOLDIN, 2006; OLIVETTI, 2013)

Aunque el proceso de migración laboral en América Latina empezó mucho antes, la globalización ${ }^{9}$ de la economía afectó la movilidad laboral en el marco de la expansión de algunos sectores económicos en detrimento de otros, produciendo un incremento de las posibilidades de acceso de la mujer a la actividad económica (RUBIN-KURTZMAN, et al., 2006), con marcadas diferencias entre países (CAMOU et al., 2011; MAUBRIGADES, 2013). La tasa de participación de las mujeres pasó de un promedio del $20 \%$ en la primera mitad del siglo XX a alcanzar cifras superiores al $40 \%$ al finalizar la segunda mitad del siglo. En particular, para la mayoría de los países de América Latina la tasa de actividad de las mujeres aumentó en forma considerable con la ola de globalización posterior a 1980. Varios autores destacan que el incremento de la demanda de mano de obra de las mujeres, está vinculado en su mayoría a trabajos de baja calificación y remuneración ${ }^{10}$ (WELLER, 1998; DELL, 2005; GÓMEZ-GALVARRIATO et al., 2005; GÓMEZ GALVARRIATO et al., 2011), en circunstancias donde los hogares no pueda sostenerse con el ingreso de un solo proveedor (BENERIA, 2001; CHANT et al., 2003; CUNNINGHAM, 2001; GONZÁLEZ DE LA ROCHA, 2000).

Las nuevas oportunidades de trabajo y la posibilidad de generar un ingreso que no existían en los lugares de origen - o también de prestaciones sociales que no tenían antes-(DENMAN, 2001), introdujeron distorsiones en la relación hombres/mujeres en las migraciones, en la composición de los hogares y en el aumento de hogares dirigidos por mujeres en los lugares de origen o de destino. Además en este período se produce una significativa disminución en la brecha en la participación entre hombres y mujeres. Esta situación resultó de la combinación de un incremento en las tasas de actividad de las mujeres, al tiempo que la actividad de los hombres, aunque se mantiene promedialmente por encima del $70 \%$, tiende a reducirse globalmente (MAUBRIGADES, 2017).

En este trabajo se realiza un análisis estadístico descriptivo de la brecha de género en la participación de los inmigrantes intrarregionales en América Latina alrededor del año 2010, poniendo en comparación la situación de la población económica nativa en los distintos mercados

la últimas dos décadas del siglo pasado, la investigación de la participación de las mujeres en el mundo del trabajo en América Latina, enfatiza la multidimensionalidad y el reconocimiento del trabajo productivo remunerado y del trabajo doméstico no-remunerado (BENERÍA, 1979; 1992; BENERÍA et al., 1987; GARCÍA et al., 1994; CHANT et al., 2003; ARIZA et al., 2003), de manera de dar visibilidad a las múltiples dimensiones del trabajo de las mujeres. No obstante estos esfuerzos por integrar el trabajo doméstico y extra-doméstico, prevalece una visión segmentada y fragmentada del trabajo femenino que, según Oliveira et al., (2000:17), "refuerza la permanente segregación de las mujeres en la dimensión desvalorizada y no remunerada del mismo: el trabajo doméstico". Mientras que Hilfinger Messias et al. (1997), rechazan esta dicotomía del trabajo femenino en esferas separadas o un continuo polarizado. La segregación ocupacional por género, una construcción histórica de la división sexual del trabajo, ha cambiado muy poco (AQUINO, 1995; DE BRITO, 1997; BUVINIC et al., 2002; CRUZ et al., 2003; LARA, 1998; MÁRQUEZ et al., 1993; OLIVEIRA et al., 2000; RAVELO BLANCAS, 1995).

${ }^{8}$ En esta misma línea, diversos autores (PAMPEL et al., 1986; PSACHAROPOULOS et al., 1989; TZANNATOS, 1999), afirman que los países con niveles más altos o más bajos de ingresos tienen mayores tasas de participación laboral de las mujeres que en los países de ingresos medios (MAUBRIGADES, 2017)

9 Entendemos la globalización como un conjunto de procesos interrelacionados que aumentan la interdependencia económica, política, social e ideológica entre países con el motivo de crear las condiciones idóneas para la producción a escala global (RUBIN-KURTZMAN, et al., 2006). Los países latinoamericanos han adoptado un modelo neoliberal dirigido hacia los mercados externos, que favoreció la inserción de las mujeres en el sector terciario y en las industrias de exportación, y ha propiciado otras transformaciones, como por ejemplo, el crecimiento del sector informal, las condiciones de trabajo precarias y la subcontratación (ARIZA et al., 2002; POLLACK, 1997; GÓMEZ GÓMEZ, 1993).

${ }^{10}$ La mayoría de los estudios sobre el trabajo femenino en América Latina se han enfocado en la situación generalizada de explotación y mala remuneración (BENERÍA, 2001). Denman (2001) sugiere que las mujeres aceptan estas condiciones desfavorables porque su motivación principal es el deseo de mejorar las condiciones de vida y asegurar la educación de sus hijos. 
nacionales. Procurando exponer el nivel de variabilidad existente en la brecha de las veinte corrientes migratorias en los diferentes países de destino dentro de la región.

Los insumos cuantitativos surgen de los resultados de los operativos censales latinoamericanos de la ronda de 2010. Además el Centro Latinoamericano y Caribeño de Demografía (CELADE), ha generado una base con información del bloque de migraciones de los países de la región correspondiente al proyecto Investigación de la Migración Internacional en Latinoamérica ${ }^{11}$ (IMILA) - . Se han llevado adelante procesos de consistencia entre ambas fuentes a los fines de aumentar el volumen de información disponible.

\section{METODOLOGÍA}

A pesar de la acelerada incorporación de la mujer a la actividad económica en las dos últimas décadas del siglo XX hasta la actualidad, y los evidentes cambios en el mercado de trabajo de América Latina, existen limitaciones para medir la inserción laboral y deficiencias en las fuentes primarias ${ }^{12}$ en la desagregación de los datos por sexo en los países de la región (POLLACK, 1997; CEPAL, 2001).

La fuerza de trabajo, fuerza laboral o población económicamente activa (PEA) ${ }^{13}$ está conformada por las personas que ofrecen su mano de obra para la producción de bienes y servicios económicos, los que corresponden al concepto de ingreso en las estadísticas de ingreso nacional. Este concepto se basa en una definición de actividad económica ambigua, no hace referencia a la producción de subsistencia y a las actividades no remuneradas -producción doméstica y tareas afines y trabajo voluntario-, realizadas mayoritariamente por mujeres ${ }^{14-15}$ (BENERÍA, 1991) tendiendo a subestimarse la fuerza de trabajo femenina-, resultando complejo de adoptar a las distintas culturas y grados de desarrollo de los países.

Cabe destacar que no todos los países de la región han realizado sus censos de población de la ronda del 2010 el mismo año, además existen algunas diferencias en los criterios para la captación de la condición de actividad ${ }^{16}$ entre los países (POLLACK, 1997), como ser el tiempo de

11 La actualización de la base es diciembre de 2015. Su información se encuentra disponible en http://www.cepal.cl/celade/migracion.

12 Todo proceso de producción estadística implica una serie de procesos específicos y relacionados entre sí, en la que participan decisiones de distintos actores, aspectos técnicos, y delimitaciones conceptuales (AGUILAR et al., 2005).

${ }^{13}$ La oferta de empleo es uno de los componentes del mercado de trabajo, y en cierto sentido el tamaño y la estructura por sexo y edad de la población “(...) determinan los límites máximos respecto del número de personas que pueden participar en la actividad económica” (ELIZAGA et al.,, 1971:13). En el mercado de trabajo se establecen relaciones entre la oferta y la demanda, sin embargo su funcionamiento tiene un carácter complejo que impiden un tratamiento similar al de los otros mercados existentes en la economía (MUÑOZ, 2009).

${ }^{14}$ Esta situación ha sido ampliamente analizada (BOSERUP, 1970; ANKER, 1987; BENERÍA, 1982 y 1991; BLADES, 1975; DIXON-MUELLER, et al. 1988; LUTZEL, 1989; WAINERMAN et al., 1981). Varias autoras han solicitado incorporar las diferentes modalidades de trabajo al análisis empírico (KERGOAT, 2003; IZQUIERDO, 1998; ACKER, 1990; LASLETT et al., 1989; RESKIN et al., 1990) y de extender la cuantificación del valor económico a las formas de trabajo no dirigidas hacia el mercado, particularmente las realizadas al interior de los hogares (PEDRERO, 2004; VANDELAC, 1994).

${ }^{15}$ En la sexta Conferencia Regional sobre la Integración de la Mujer en el Desarrollo Económico y Social de América Latina y el Caribe de 1994, se solicita que se generalice la desagregación por sexo en las estadísticas, así como para incluir en ellas indicadores de género y etnia, y favorecer la incorporación del análisis diferencial en función del género en los sistemas estadísticos (CEPAL, 1995).

${ }^{16}$ La edad mínima de la población y preguntas agrupadas utilizadas para determinar la condición de actividad presenta ciertas diferencias en algunos países. La consistencia de la participación en la actividad económica se realizado para la población de 14 años y más. La PEA quedó clasificada en una sola de estas categorías: ocupados, desocupados e inactivos. Dando prioridad a las actividades en empleo sobre las de búsqueda de empleo y a estas últimas respecto de cualquier actividad no remunerada. Clasificando como empleado a quien trabaja y busca otro empleo a la vez, mientras que se clasificó como desempleado a un estudiante que al mismo tiempo que prosigue sus estudios trata de conseguir un empleo (PANIGO et al., 2014). 
referencia para considerar una persona empleada ${ }^{17}$, tal como puede apreciarse en la Figura 1. Debido a lo cual, debieron realizarse acciones para asegurar la consistencia y comparabilidad entre los datos de diferentes países. Las personas en edad de trabajar quedaron divididas, en las que se encuentren trabajando o deseen trabajar-PEA-, y población restante que no participa de la actividad económica. Siendo la tasa de actividad el indicador que cuantifica el nivel de participación de la PEA. Su cálculo resulta del cociente entre la PEA y la población en edad de trabajar, expresado en términos porcentuales (MANZANO et al., 2016). Este instrumento resulta útil para analizar las desigualdades de género, dado que el mercado de trabajo es un espacio que se destaca por la persistente desigualdad entre sexos (MIKKOLA, 2005).

Los censos de población aportan información del saldo de la migración neta ${ }^{18}$ de un país durante el periodo intercensal -el stock de personas que han migrado entre dos momentos del tiempo- (MARTÍNEZ PIZARRO, 2003). Esto se logra aplicando preguntas sobre la ubicación de las personas en una fecha previa al operativo censal -en la mayor parte de los casos se indaga sobre el país de nacimiento y el lugar de residencia 5 años atrás-. Por consiguiente, se supone que durante ese periodo de tiempo sobre el cual hace referencia el censista, estas personas no han reemigrado, ni vuelto a su país de origen. Pese a estas limitaciones ${ }^{19}$, las potencialidades que surgen de los microdatos del censo siguen siendo fuertes para obtener una aproximación de las características y repercusiones de la migración internacional (VILLA et al., 2002).

Las cantidad preguntas correspondientes al bloque de migraciones en las cedulas censales no son exactamente las mismas en cada uno de los países de la región (CELADE, 2014).

\footnotetext{
${ }^{17}$ Resulta más probable una persona esté empleada por un corto período de tiempo que por uno largo, siendo las mujeres más afectadas que los varones por los empleos temporales, por tanto la magnitud de la cantidad de personas ocupadas según sexo puede verse afectada (POLLACK, 1997).

${ }^{18}$ Los resultados que surgen permiten conocer la cantidad de migrantes pero no de las migraciones. No relevan la cantidad de ingresos y salidas que realizó un migrante internacional en un país durante el periodo intercensal -flujo migratorio bruto-, como tampoco indagan los retornos a la población de origen, ni la incidencia de la mortalidad (FAURA MARTÍNEZ et al., 2002).

${ }^{19}$ Se carece de una definición universal de la migración (SHAW, 1975), así como tampoco existe una homogeneidad conceptual ni metodológica dentro de la amplia y variada bibliografía relacionada a su estudio (FAURA MARTÍNEZ et al., 2002), teniendo su correlato en las dificultades para su registro y medición.
} 
FIGURA 1

Preguntas referidas a la actividad económica de las personas en los censo de los países latinoamericanos alrededor del año 2010.

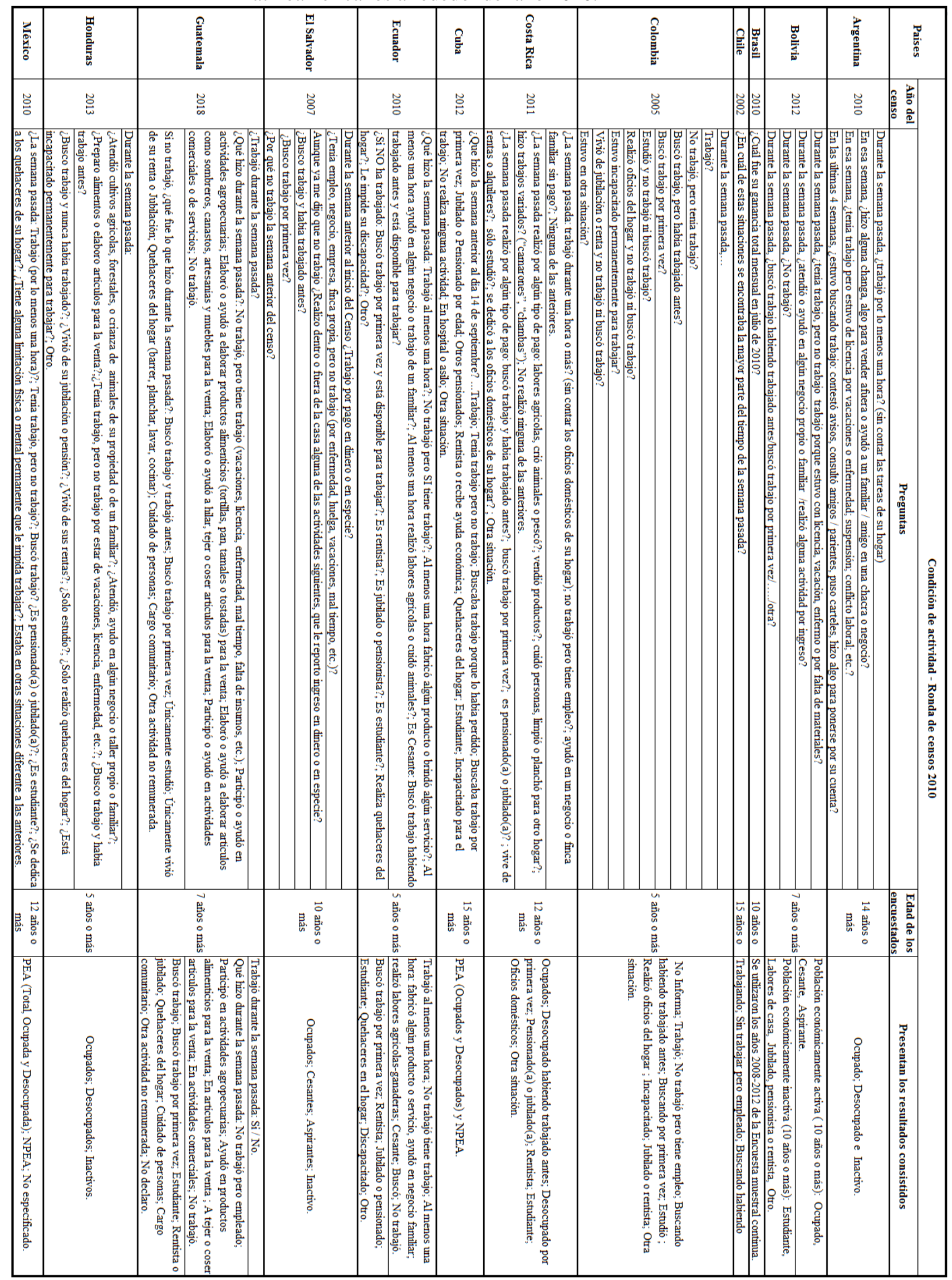







Fuente: Elaboración personal en base a información de los institutos estadísticos de los países ${ }^{20}$.

\section{RESULTADOS}

Alrededor del año 2010 América Latina tenía una población de más de 557 millones de personas, con un índice de masculinidad -en adelante I.M.- de 96, $2^{21}$. La distribución espacial de la población de la región se destaca por una marcada desigualdad, el $68,7 \%$ de la población masculina y el 69,1\% de la femenina residían en solo cuatro países-Brasil, México, Colombia y Argentina-

Existían un total de 6,6 millones de extranjeros residiendo en países latinoamericanos ${ }^{22}$, siendo en este caso 99,3 el número de varones por cada 100 mujeres. También fuertemente concentrados, el $66,9 \%$ de los varones y $69,2 \%$ de las mujeres aglomerados en cuatro destinos -en orden descendente Argentina, Venezuela, México y Brasil-.

La composición migratoria resultaba 64,2\% para los extranjeros nacidos en América Latina, con un mayor predominio femenino, siendo el valor de I.M. de 95,8, y el 35,8\% de los inmigrantes restantes nacidos fuera de la región, destacándose este contingente por una mayor presencia del sexo masculino, registrándose 106,1 varones por cada 100 mujeres en los años cercanos al $2010^{23}$.

De la información presente en la Tabla 1, pueden diferenciarse: aquellos países con población extranjera -nacido adentro y fuera de la región- con mayor presencia femenina que la nativa Argentina, Cuba, Uruguay y Venezuela-, resultando la población total aún más feminizada; un segundo grupo de países con población extranjera con predominio masculino respecto a la nativa ${ }^{24}$-Bolivia, Brasil, Colombia, Ecuador, Nicaragua, Paraguay y República Dominicana-, derivando en una población total con mayor presencia masculina ${ }^{25}$; y los restantes países que presentaron una población nativa con un I.M. mayor al de los inmigrantes nacidos en la región e inferior a los extranjeros extrarregionales -Chile, Costa Rica, El Salvador, Guatemala, Honduras, México, Panamá y Perú-, los elevados I.M. de los viejos inmigrantes de ultramar compensa los menores I.M. de los contingentes de inmigrantes latinoamericanos ${ }^{26}$-a pesar que el peso relativo

\footnotetext{
${ }^{20}$ El Instituto Haitiano de Estadística y Tecnología de la Información (IHSI), cuenta con tres censos de población realizados a diferentes intervalos $(1950,1971,1982)$ (CELADE, 1991). El cuarto realizado en 2003 - más de veinte años después del de 1982-, quedó obsoleto tras el terremoto que devastó al país en 2010 (RESERVE, 2014). En el año 2018 el IHSI realizado su último censo de población y vivienda, con apoyo de organismos internacionales. Debido a esta situación solo se poseen los datos de los emigrantes haitianos captados en los censos de los restantes países de América Latina en fechas alrededor del año 2010. ${ }^{21}$ El índice de masculinidad es la razón entre varones y mujeres de una población multiplicada por cien, muestra el número de varones por cada 100 mujeres.

${ }^{22} \mathrm{La}$ variabilidad de la presencia extranjera en los países tiene una elevada variabilidad -siendo el coeficiente de variación de un valor de $110,7 \%-$, se destacan por la mayor participación relativa de inmigrantes: Costa Rica con 9,0\% de la población total, Argentina 4,5\%, Panamá 4,3\%, Venezuela y República Dominicana 4,2\% y Paraguay 3,8\%.

${ }^{23}$ Los extranjeros nacidos fuera de la región se encuentran más concentrados que los propios de la región. En el primer caso el $77,9 \%$ de los varones y 78,9\% de las mujeres en tan solo cuatro países -México, Brasil, Argentina, y Venezuela-, y en el segundo, 72,3\% de los varones de los inmigrantes latinoamericanos y $72,9 \%$ de las mujeres en cuatro destinos - en orden descendente Argentina, Venezuela, República Dominicana y Costa Rica-.

${ }^{24}$ Se destaca el caso de Uruguay, siendo la segunda población nativa más feminizada (I.M. 92,8), la inmigración residente presentó una mayor proporción de mujeres (I.M. 82,5).

${ }^{25}$ República Dominicana poseía una población nativa con mayor presencia femenina (I.M. 97,3), el componente extranjero con alta mayoría de varones y el elevado peso relativo del mismo, generó una población total con predominio masculino (I.M. 100,7). La población nativa de Paraguay era la segunda con mayor presencia masculina de la región -alrededor del año 2010-, con el aporte de la población extranjera todavía más masculinizada, en consecuencia su población total tuvo el máximo valor en el I.M. $(101,7)$.

${ }^{26}$ Dada la alta presencia de la población extranjera en Costa Rica $-7,9 \%$ de la población total eran extranjeros nacidos en Latinoamérica y 0,9\% fuera de la región-, y en Panamá -la población total estaba compuesto por $2,5 \%$ de inmigrantes intrarregionales y $1,9 \%$ de extranjeros nacidos en otras partes-, los valores de I.M. lograron retroceder 1,1 y 1,0 puntos porcentuales, respectivamente.
} 
de estos últimos es mayor, con excepción de los casos de México y Perú-.

FIGURA 2

Índice de masculinidad, población e inmigrantes en los países latinoamericanos alrededor del año 2010 (estimaciones en miles de personas).



Fuente: Elaboración personal en base a datos de los censos de población de los países de América Latina y del Proyecto IMILA del CELADE.

Alrededor del año 2010, en base a los censos realizados en América Latina, el diferencial de tasas de partición laboral según sexo de la población nativa a nivel regional (Contartese et. al, 2005), tuvo un valor de 32,4 puntos porcentuales -en adelante p.p.-, y en la población extranjera latinoamericana fue algo inferior $-29,6$ p.p.-.

Considerando a nivel país, la brecha de género más elevada en los nativos se produjo en Nicaragua con 69,7 p.p., y la menor en Uruguay de 18,3 p.p. - siempre a favor de los hombres-. En el caso de los inmigrantes intrarregionales el valor máximo de la brecha de género se dio en República Dominicana con un valor de 48,9 p.p. y el mínimo en Cuba con 12,3 p.p., siendo el rango inferior a la población nativa.

La dispersión en la brecha de género de las tasas de actividad -medida a través del coeficiente de variación, en adelante C.V.-, en los nativos fue de 34,8\% y en los extranjeros nacidos en la región de 35,5\%. La relación lineal en la brecha de género de la tasa de participación-medida a través del coeficiente de correlación " $\mathrm{R}^{2}$ "-, entre los nativos y los migrantes nacidos en la región en los distintos mercados de trabajo es nula -un valor de solo 0,11 (ver Tabla 1)-. En atención a lo mencionado, cabe destacar las elevadas diferencias en las brechas de género entre nativos y extranjeros latinoamericanos en los mercados laborales de Chile, Cuba, y Nicaragua, con superioridad por parte de los primeros, y los casos de Paraguay, República Dominicana y Venezuela, con una mayor brecha en los inmigrantes intrarregionales. 
TABLA 1

Tasa de actividad en los mercados laborales nacionales y de las corrientes migratorias según sexo. Países de América Latina en fechas alrededor del año 2010.

\begin{tabular}{|c|c|c|c|c|c|c|c|}
\hline \multicolumn{8}{|c|}{ Tasa de actividad en los mercados nacionales } \\
\hline Población nativa & Varón & Mujer & $\begin{array}{l}\text { Brecha } \\
\text { de } \\
\text { generó }\end{array}$ & $\begin{array}{l}\text { Extranjeros/as } \\
\text { nacidos en la } \\
\text { región }\end{array}$ & Varón & Mujer & $\begin{array}{l}\text { Brecha } \\
\text { de } \\
\text { generó }\end{array}$ \\
\hline América Latina & 67,1 & 34,7 & 32,4 & América Latina & 82,1 & 52,5 & 29,6 \\
\hline Argentina (2010) & 77,4 & 54,6 & 22,7 & Argentina & 84,7 & 62,1 & 22,6 \\
\hline Bolivia(2012) & 69,9 & 49,7 & 20,2 & Bolivia & 54,2 & 34,4 & 19,7 \\
\hline Brasil (2010) & 73,2 & 50,7 & 22,5 & Brasil & 77,8 & 55,7 & 22,1 \\
\hline Chile (2002) & 70,0 & 35,6 & 34,5 & Chile & 70,4 & 52,6 & 17,8 \\
\hline Colombia (2005) & 53,4 & 28,0 & 25,4 & Colombia & 52,7 & 30,7 & 22,0 \\
\hline Costa Rica (2011) & 66,0 & 32,9 & 33,1 & Costa Rica & 80,3 & 41,3 & 39,0 \\
\hline Cuba (2012) & 90,0 & 60,5 & 29,5 & Cuba & 50,5 & 38,2 & 12,3 \\
\hline Ecuador (2010) & 59,9 & 34,0 & 25,8 & Ecuador & 76,7 & 46,6 & 30,2 \\
\hline E1 Salvador (2007) & 58,3 & 34,3 & 24,0 & E1 Salvador & 71,8 & 38,7 & 33,1 \\
\hline Guatemala (2018) & 59,5 & 23,4 & 36,1 & Guatemala & 74,2 & 34,0 & 40,2 \\
\hline Honduras (2013) & 55,3 & 17,8 & 37,5 & Honduras & 67,6 & 24,2 & 43,4 \\
\hline México (2010) & 74,1 & 33,5 & 40,6 & México & 85,4 & 41,7 & 43,7 \\
\hline Nicaragua (2005) & 97,2 & 27,5 & 69,7 & Nicaragua & 59,3 & 29,1 & 30,2 \\
\hline Panamá (2010) & 66,4 & 36,5 & 29,9 & Panamá & 75,1 & 50,4 & 24,7 \\
\hline Paraguay (2002) & 68,6 & 32,8 & 35,8 & Paraguay & 82,1 & 34,9 & 47,2 \\
\hline Perú (2007) & 58,0 & 31,1 & 26,9 & Perú & 50,1 & 27,8 & 22,3 \\
\hline R. Dominicana (201 & 67,5 & 36,3 & 31,2 & R. Dominicana & 88,3 & 39,4 & 48,9 \\
\hline Uruguay (2011) & 69,8 & 51,4 & 18,3 & Uruguay & 76,6 & 54,8 & 21,9 \\
\hline Venezuela (2011) & 79,7 & 50,6 & 29,2 & Venezuela & 81,4 & 39,4 & 41,9 \\
\hline c.v. & 16,0 & 29,7 & 34,9 & c.v. & 16,9 & 24,8 & 35,5 \\
\hline $\mathbf{R}^{2}$ & - & 0,28 & - & $\mathbf{R}_{\text {Varón, Mujer }}^{2}$ & - & 0,28 & - \\
\hline & & & & $\mathbf{R}_{\text {Nativos, Extranj. }}^{2}$ & 0,00 & 0,36 & 0,11 \\
\hline
\end{tabular}

Fuente: Elaboración personal en base a datos de los censos de población de los países de América Latina y del Proyecto IMILA del CELADE.

Comparando las brechas de género ${ }^{27}$ en las tasas de actividad entre la población extranjera en los distintos mercados nacionales, el valor máximo fue de 72,8 p.p. correspondiente a la corriente migratoria costarricense residente en México, mientras que en este mismo mercado laboral la mano de obra uruguaya presenta una brecha de tan solo 5,0 p.p. Adicionalmente la fuerza de trabajo costarricense exhibe una brecha de 11,4 y 15,0 p.p. en los mercados nacionales de República Dominicana y Colombia, respectivamente. Por tanto, existe una amplia variabilidad en la brecha tanto al interior de los mercados de trabajo de los países, como de las corrientes migratorias. En el primer caso se destacan Cuba, Paraguay y Nicaragua -los valores de C.V. fueron $67,1,61,2 \mathrm{y} 45,7 \%$, respectivamente-, y en las corrientes migratorias la paraguaya $(59,6 \%)$, dominicana $(55,9 \%)$ y haitiana $(54,8 \%)$ (ver Tabla 2$)$.

En relación con lo mencionado, se destaca que la relación lineal entre los coeficientes de variaciones de los mercados de trabajo y las corrientes migratorias, es nula - un valor de $\mathrm{R}^{2} \mathrm{de}$ solo $0,03-$.

\footnotetext{
${ }^{27}$ Se fijó como límite mínimo una cantidad de población extranjera en edad de trabajar superior a las 50 personas en cada mercado de trabajo nacional, a nivel total y según sexo.
} 
TABLA 2

Brecha de género ${ }^{28}$ en la tasa de actividad según país de nacimiento. Países de América Latina en fechas alrededor del año 2010.

\begin{tabular}{|c|c|c|c|c|c|c|c|c|c|c|c|c|c|c|c|c|c|c|c|c|}
\hline$\because$ & 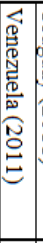 & & 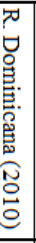 & $\begin{array}{ll}0 \\
0 \\
0\end{array}$ & & 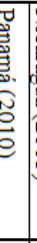 & & 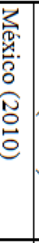 & 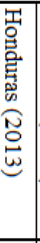 & 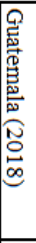 & 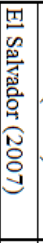 & 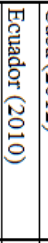 & & 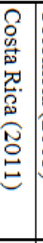 & 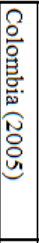 & 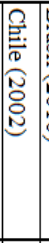 & & & & 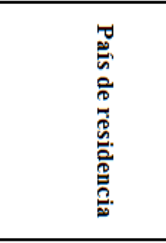 \\
\hline & $\begin{array}{l}\vec{*} \\
0 \\
0\end{array}$ & $\underset{\sim}{\sim}$ & $\begin{array}{c}\vec{\infty} \\
0 \\
0\end{array}$ & 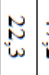 & $\begin{array}{c}\vec{N} \\
\mathrm{~N}\end{array}$ & s. & 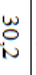 & 岕 & $\begin{array}{c}\vec{\omega} \\
\stackrel{+}{*}\end{array}$ & $\underbrace{}_{\substack{0 \\
\text { N }}}$ & $\mid \begin{array}{c}w \\
w \\
-\end{array}$ & $\mid \begin{array}{l}w \\
0 \\
\text { on } \\
\end{array}$ & $\vec{w}$ & $\begin{array}{l}\omega \\
0 \\
0 \\
0\end{array}$ & N & 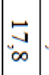 & 点 & $\begin{array}{l}\overrightarrow{0} \\
ن \\
v\end{array}$ & $\begin{array}{l}n \\
\text { Na }\end{array}$ & Latinoamérica \\
\hline $\mid \begin{array}{c}n \\
\infty \\
\infty \\
\infty\end{array}$ & $\mid$ & $\vec{\infty}$ & N & {$\left[\begin{array}{ll}\vec{n} \\
\infty \\
\infty\end{array}\right]$} & $\begin{array}{c}\mathfrak{c} \\
\infty \\
a\end{array}$ & $\begin{array}{c}w \\
\alpha \\
\infty \\
\infty\end{array}$ & & $\begin{array}{c}w \\
. \\
\sigma \\
\sigma\end{array}$ & N & $\begin{array}{l}N \\
\infty \\
a \\
a\end{array}$ & $\begin{array}{l}\stackrel{\bullet}{\infty} \\
\circ \\
0\end{array}$ & 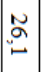 & & $\begin{array}{l}n \\
+ \\
0 \\
0\end{array}$ & 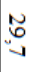 & 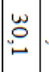 & $\stackrel{\mathrm{s}}{\mathrm{I}}$ & $\begin{array}{l}\vec{w} \\
\vdots \\
0\end{array}$ & N & Argentina \\
\hline$\because$ & $\left(\begin{array}{c}N \\
+ \\
N\end{array}\right.$ & $\begin{array}{l}N \\
N \\
0\end{array}$ & $\stackrel{N}{\circ}$ & $\stackrel{\sim}{\sim}$ & مू & $\begin{array}{l}\vec{v} \\
\dot{v}\end{array}$ & & 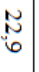 & & & & \begin{tabular}{c}
$\tilde{O}$ \\
$\sim$ \\
\hdashline
\end{tabular} & & $\begin{array}{l}n \\
0 \\
\infty \\
\infty\end{array}$ & $\vec{N}$ & $\mid$ & $\overrightarrow{\breve{a}}$ & $\begin{array}{c}\text { No } \\
\text { is. }\end{array}$ & ڤ̆ & Bolivia \\
\hline$\stackrel{+}{+}$ & $\begin{array}{c}w \\
\infty \\
\infty \\
\infty\end{array} \mid$ & 茫 & $\underset{\omega}{\omega}$ & $\mid \begin{array}{l}\tilde{y} \\
\ddot{0} \\
0\end{array}$ & : & 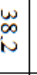 & & $\begin{array}{c}\overrightarrow{0} \\
\text { is } \\
\end{array}$ & $\begin{array}{l}0 \\
+ \\
\end{array}$ & 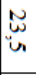 & $\vec{a}$ & 芯 & & \begin{tabular}{l}
$\sim$ \\
$\stackrel{\circ}{\sim}$ \\
\hdashline
\end{tabular} & 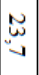 & $\mid \begin{array}{c}\vec{\omega} \\
\stackrel{\sim}{N}\end{array}$ & $\begin{array}{c}n \\
N \\
i n\end{array}$ & $\begin{array}{l}\vec{a} \\
\vec{u}\end{array}$ & N & Brasil \\
\hline N & $\begin{array}{l}\omega \\
\vec{\omega} \\
\end{array}$ & $\vec{w}$ & 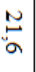 & $\mid \begin{array}{c}\tilde{N} \\
\alpha \\
i\end{array}$ & $\stackrel{\omega}{\omega}$. & $\begin{array}{l}w \\
0 \\
0 \\
0\end{array}$ & & $\begin{array}{l}\vec{w} \\
i \\
w\end{array}$ & 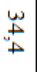 & & $\begin{array}{l}N \\
\infty \\
i n \\
ن\end{array}$ & $\begin{array}{l}n \\
\dddot{n} \\
a\end{array}$ & $\begin{array}{l}u_{u} \\
\ddot{v}\end{array}$ & $\begin{array}{c}\mathfrak{M} \\
\infty \\
\infty\end{array}$ & 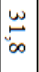 & $\mid \begin{array}{c}w \\
\underset{w}{w}\end{array}$ & 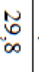 & 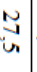 & $\tilde{\omega}$ & Chile \\
\hline$\underset{\sim}{\stackrel{\sim}{\sim}}$ & $\underset{\omega}{ \pm}$ & $\vec{w}$ & $\underset{\omega}{\vec{w}}$ & $\mid \begin{array}{c}N \\
\vec{w} \\
w\end{array}$ & $\begin{array}{l}\mathfrak{0} \\
0 \\
0\end{array}$ & 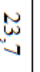 & & 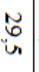 & : & $\omega$ & $\begin{array}{l}N \\
N \\
\infty\end{array}$ & $\begin{array}{l}\text { w } \\
\stackrel{\circ}{\sim}\end{array}$ & & 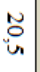 & \begin{tabular}{l}
$n$ \\
$\sim$ \\
\hdashline \\
\hdashline
\end{tabular} & $\mid \begin{array}{l}\vec{\infty} \\
\dot{w} \\
\ddot{*}\end{array}$ & 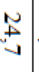 & 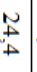 & $\vec{\omega}$ & Colombia \\
\hline 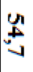 & $\begin{array}{l}w \\
N \\
N \\
N\end{array}$ & & $\begin{array}{l}\vec{\Xi} \\
{ }_{\Delta}\end{array}$ & & & $\begin{array}{c}w \\
\underset{+}{w} \\
-\end{array}$ & $\begin{array}{c}N \\
\infty \\
0 \\
0\end{array}$ & $\begin{array}{c}\mathbf{N} \\
\infty \\
\infty\end{array}$ & 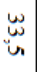 & 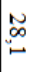 & $\begin{array}{l}N \\
N \\
0\end{array}$ & \begin{tabular}{l}
$N$ \\
$\circ$ \\
\hdashline \\
+
\end{tabular} & & $\underset{\sim}{\sim}$ & $\vec{n}$ & & & & E & Costa Rica \\
\hline$\underset{\sim}{\stackrel{N}{N}}$ & $\infty \begin{array}{l}\infty \\
\infty\end{array}$ & 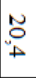 & $\stackrel{\infty}{-}$ & $\begin{array}{l}\mathbf{W} \\
N \\
\infty \\
\infty\end{array}$ & $\stackrel{\infty}{\infty}$ & $\begin{array}{l}\tilde{N} \\
0 \\
0\end{array}$ & $\begin{array}{c}\omega \\
\omega \\
+ \\
+\end{array}$ & 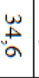 & 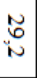 & $\overrightarrow{0}$ & $\begin{array}{l}N \\
\curvearrowright \\
\alpha\end{array}$ & 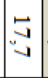 & 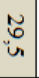 & $\stackrel{\sim}{\sim}$ & $\begin{array}{l}n \\
\infty \\
\infty \\
N \\
N\end{array}$ & $\begin{array}{l}\vec{b} \\
\dot{u}\end{array}$ & 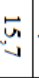 & 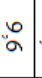 & $\frac{\omega}{+}$ & Cuba \\
\hline$\underset{\infty}{\mathbf{W}}$ & {$\left[\begin{array}{l}w \\
\tilde{N} \\
0\end{array}\right.$} & $\infty$ & 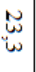 & $\left|\begin{array}{l}w \\
\sim \\
\sim \\
\sim\end{array}\right|$ & & $\begin{array}{l}\omega_{\circ}^{\prime} \\
\circ\end{array}$ & & $\underset{\sim}{ \pm}$ & & & $\begin{array}{l}w \\
u \\
\sim \\
\sim\end{array}$ & $\begin{array}{l}N \\
\dddot{n} \\
\infty\end{array}$ & & $\stackrel{\sim}{ \pm}$ & $\mid \begin{array}{l}0 \\
0 \\
a \\
a\end{array}$ & $\mid \begin{array}{l}\vec{u} \\
\ddot{n}\end{array}$ & $\stackrel{\sim}{\sim}$ & 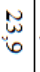 & 二 & Ecuador \\
\hline ్ֶ & $\mid$ & & $\begin{array}{l}w \\
\vec{t} \\
0\end{array}$ & & & 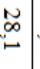 & $\begin{array}{c}w \\
\sim \\
\sim \\
\sim\end{array}$ & $\begin{array}{c}+\infty \\
\sim \\
\sim\end{array}$ & 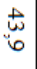 & $\begin{array}{l}\omega \\
\text { à } \\
0\end{array}$ & $\begin{array}{l}n \\
+ \\
0\end{array}$ & & & 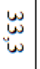 & $\begin{array}{l}n \\
t \\
t\end{array}$ & & & & a. & El Salvador \\
\hline 崩 & & & న్ & & & $\begin{array}{c}N \\
\substack{n \\
\infty}\end{array}$ & 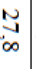 & $\begin{array}{l}u \\
\vec{a}\end{array}$ & $\begin{array}{l}\vec{a} \\
\stackrel{a}{+}\end{array}$ & $\stackrel{\omega}{\omega}$ & $\mid \begin{array}{l}\omega \\
w \\
\omega\end{array}$ & & & $\underset{\sim \sim \sim}{\sim}$ & N & & & & 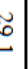 & Guatemala \\
\hline$\underset{\substack{u \\
+\infty \\
+\infty}}{4}$ & $\left.\begin{array}{c}\infty \\
\infty \\
\infty \\
\infty\end{array}\right]$ & & $\begin{array}{l}u_{0} \\
\text { o } \\
\infty\end{array}$ & & & $\begin{array}{l}\mathfrak{N} \\
\stackrel{0}{0} \\
\sim \\
\end{array}$ & & & & & & $\mid \begin{array}{l}\vec{n} \\
\overrightarrow{+}\end{array}$ & $\stackrel{N}{\sim}$ & \begin{tabular}{l}
\multirow{2}{*}{} \\
$\stackrel{\circ}{\circ}$
\end{tabular} & & & & & N & Haití \\
\hline$\omega_{\infty}^{\omega}$ & & & 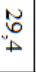 & & & 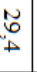 & & $\begin{array}{l}u \\
\vec{t} \\
a \\
a\end{array}$ & $\begin{array}{l}\vec{u}^{\prime} \\
\text { un }\end{array}$ & $\underset{\omega}{\omega}$ & $\begin{array}{l}w \\
w \\
w \\
\omega\end{array}$ & & & 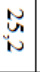 & $\underset{N}{\sim}$ & & & & $\infty$ & Honduras \\
\hline जू & $\begin{array}{l}w \\
u \\
\vdots \\
r\end{array}$ & 垈 & $\begin{array}{l}0 \\
\infty \\
0 \\
0\end{array}$ & $\left|\begin{array}{c}\vec{\infty} \\
\infty \\
\omega\end{array}\right|$ & $\begin{array}{l}n \\
0\end{array}$ & $\begin{array}{l}\tilde{\omega} \\
\infty \\
\infty\end{array}$ & \begin{tabular}{l}
$\vec{u}$ \\
\hdashline \\
$\sim$
\end{tabular} & $\begin{array}{c}\overrightarrow{0} \\
\text { is }\end{array}$ & 芯 & un & $\begin{array}{l}\overrightarrow{0} \\
\circ\end{array}$ & $\stackrel{N}{\sim}$ & $\ddot{b}$ & 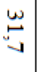 & $\stackrel{\bullet}{\sim}$ & $\begin{array}{l}\sim \\
\dddot{N} \\
\sim\end{array}$ & 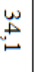 & & 䒧 & México \\
\hline$\frac{5}{6}$ & $\begin{array}{l}\tilde{0} \\
0 \\
\cdots\end{array}$ & & $\underset{\sim}{\vec{w}}$ & & & $\begin{array}{l}\vec{b} \\
\stackrel{\vec{v}}{*}\end{array}$ & 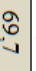 & 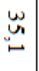 & $\begin{array}{c}\infty \\
\infty \\
\infty \\
\infty\end{array}$ & $\stackrel{\vec{N}}{*}$ & $\begin{array}{l}\omega \\
N \\
0\end{array}$ & & $\begin{array}{l}\omega \\
. \\
0\end{array}$ & 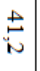 & $\vec{\infty}$ & & & & $\stackrel{\sim}{\vec{t}}$ & Nicaragua \\
\hline $\begin{array}{l}+\infty \\
0 \\
0\end{array}$ & $\mid \begin{array}{c}\tilde{u} \\
w \\
w\end{array}$ & & $\underset{w}{w}$ & & & 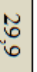 & & \begin{tabular}{l}
$\mathbb{0}$ \\
$\stackrel{0}{0}$ \\
\hdashline
\end{tabular} & $\stackrel{\sim}{\sim}$ & 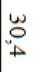 & $\begin{array}{l}\tilde{w} \\
\omega \\
0\end{array}$ & & & $\begin{array}{l}u \\
\text { ù } \\
\text { o. }\end{array}$ & $\begin{array}{l}\vec{u} \\
\vec{p}\end{array}$ & $\Xi$ & $\stackrel{\infty}{i}$ & & $\vec{n}$ & Panamá \\
\hline 경 & & $\underset{\sim}{\omega}$ & & & $\begin{array}{c}w \\
w \\
\text { s. } \\
\infty\end{array}$ & & & $\stackrel{\text { ò }}{\mathrm{a}}$ & & & & & & & $\stackrel{\infty}{\sim}$ & $\overrightarrow{+}$ & 芯 & $\begin{array}{c}\omega \\
\stackrel{\omega}{\Delta} \\
\sim\end{array}$ & $\begin{array}{l}n \\
\vdots \\
a\end{array}$ & Paraguay \\
\hline 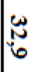 & $\mid \begin{array}{l}w \\
N \\
0 \\
0\end{array}$ & 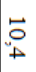 & $\begin{array}{c}n \\
\stackrel{+}{0}\end{array}$ & $\mid \begin{array}{l}n \\
\alpha \\
0\end{array}$ & . & $\begin{array}{c}\omega \\
\vec{\sigma} \\
\sigma\end{array}$ & & $\overrightarrow{\vec{w}}$ & $\stackrel{\omega}{\omega}$ & $\underbrace{w}_{u}$ & $\underset{\omega}{\omega}$ & $\begin{array}{l}w \\
\vec{J} \\
u\end{array}$ & & 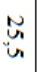 & טֵ & $\begin{array}{l}\overrightarrow{0} \\
\infty \\
\infty\end{array}$ & 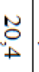 & $\begin{array}{c}n \\
\text { and } \\
a\end{array}$ & $E$ & Perú \\
\hline 学 & $\begin{array}{l}\omega \\
\omega \\
\omega\end{array} \mid$ & & $\underset{\sim}{\vec{w}}$ & & & $\begin{array}{c}\overrightarrow{\vec{u}} \\
\mathrm{u}_{i}\end{array}$ & & $\stackrel{2}{3}$ & 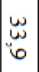 & & & & & 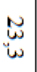 & $\ddot{\alpha}$ & & & & $\vec{\omega}$ & R. Dominicana \\
\hline $\begin{array}{l}\omega \\
0 \\
0 \\
0\end{array}$ & $\begin{array}{l}\omega \\
\vec{\omega} \\
\dot{\omega}\end{array}$ & $\underset{\substack{\infty \\
\infty}}{\infty}$ & 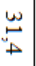 & $\begin{array}{l}\omega \\
\stackrel{\omega}{\omega} \\
\sim\end{array}$ & & $\begin{array}{c}w \\
\stackrel{w}{ت}\end{array}$ & & $\ddot{0}$ & & & & \begin{tabular}{l}
$N$ \\
$\infty$ \\
\hdashline
\end{tabular} & & $\begin{array}{c}N \\
\substack{\infty \\
\sim \\
\sim}\end{array}$ & 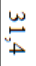 & \begin{tabular}{l}
$\sim$ \\
\hdashline \\
0
\end{tabular} & مa & & 等 & Uruguay \\
\hline 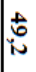 & $\left|\begin{array}{l}\tilde{N} \\
0 \\
i \\
\tilde{N}\end{array}\right|$ & $\vec{\forall}$ & $\stackrel{\infty}{-}$ & : & $\stackrel{\circ}{\circ}$ & 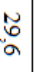 & & $\begin{array}{l}\vec{a} \\
\infty \\
\infty\end{array}$ & & $\vec{\Delta}$ & $\underset{\sim}{N}$ & $\overrightarrow{0}$ & & $\begin{array}{l}N \\
N \\
\infty \\
\infty\end{array}$ & $\vec{v}$ & $\because 0$ & $\begin{array}{l}N \\
N \\
w\end{array}$ & & 5 & Venezuela \\
\hline & 离 & 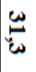 & $\stackrel{\omega}{\Delta}$ & $\mid \begin{array}{l}n \\
\infty \\
\infty \\
\infty\end{array}$ & 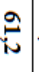 & 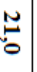 & v & 岕 & 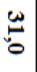 & $\begin{array}{l}n \\
\infty \\
0 \\
b\end{array}$ & $\stackrel{N}{N}$ & $\begin{array}{c}n \\
\infty \\
\infty \\
\infty\end{array}$ & $\because$ & $\vec{u}$ & $\stackrel{\omega}{\omega}_{\omega}^{\omega}$ & 壳 & ") & 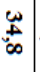 & & 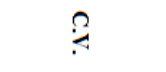 \\
\hline
\end{tabular}

Fuente: Elaboración personal en base a datos de los censos de población de los países de América Latina y del Proyecto IMILA del CELADE.

\footnotetext{
${ }^{28}$ Los datos de tasa de actividad según sexo se encuentran en el apartado Anexo (Tablas 3 y 4 ).
} 


\section{DISCUSIÓN}

La teoría migratoria consideraba la decisión de migrar como algo bajo dominio masculino, presumiendo la migración femenina como no autónoma (LIPSZYC, 2004, p.66). Recién en la segunda mitad del siglo XX se comenzó a reflejar un avance de la feminización en las corrientes migratorias mediante el uso de datos cuantitativos ${ }^{29}$ (COURTIS et al., 2010).

Autores como Oliveira y Ariza (2000) detallan cómo la combinación de la división sexual del trabajo al interior del hogar y el aumento de actividad extra-doméstica constituyen los ejes principales de desventaja económica ${ }^{30}$ y social relativa, o de exclusión social de las mujeres, vinculado a existencia de una fuerte segmentación ocupacional ${ }^{31}$ por sexo (ANKER, 1998).

Desde el lado de la demanda del mercado de trabajo, el sector servicios se expande y su peso relativo comienza a ser mayoritario en la generación de puesto de trabajo en el conjunto de la economía, al tiempo que las mujeres han mejorado su nivel educativo ${ }^{32}$ y han logrado balancear sus responsabilidades domésticas con las laborales, de modo que se abre una ventana de oportunidad en la que el ingreso de las mujeres al mercado de trabajo parece acelerarse (SEGUINO, 2000; CAMOU et al., 2011; GADDIS et al., 2013, 2014). Adicionalmente la ideología dominante supone que las mujeres están más dispuestas a aceptar condiciones laborales desfavorables y sueldos inferiores (HARLOW et al., 2004).

No obstante las investigaciones que han tratado de estimar las desigualdades de género, sostienen que la brecha de género tiende a reducirse en el tiempo conforme aumenta el nivel de riqueza de las sociedades (COLLIER 1993, DÓLAR et al.,1999, FORSYTHE et al. 2000).

Del análisis realizado surge que la brecha de género en la tasa de participación de la población nativa más elevada se produjo en Nicaragua, y la segunda mayor en México, sin embargo el ingreso per cápita en el año 2010 era de solo 3.914 dólares $^{33}$ en el primer caso, y 14.276 dólares en el último (MADDISON PROJECT DATABASE, 2018). Además se revela la ausencia de relación lineal en la brecha de género de la tasa de actividad en los distintos mercados de trabajo nacionales entre los trabajadores nativos y los migrantes nacidos en la región. En sintonía con la mirada de Gaddis y Klasen (2013), el vínculo entre la tasa de actividad de las mujeres y el nivel de desarrollo económico es más complejo de lo que supone una parte de la literatura empírica existente $^{34}$.

En las últimas décadas el aumento de la oferta laboral femenina en la región fue inédito,

${ }^{29}$ La decisión de migrar no es solo económica, se trata de un fenómeno social y cultural, en donde intervienen múltiples factores interrelacionados. Se carece de una definición universal de la migración (SHAW, 1975), así como tampoco existe una homogeneidad conceptual ni metodológica dentro de la amplia y variada bibliografía relacionada a su estudio (FAURA MARTÍNEZ et al., 2002), teniendo su correlato en las dificultades para su registro y medición.

${ }^{30}$ Considerando que hombres y mujeres tienen capacidades homogéneas, existe una menor demanda de trabajo femenino respecto al masculino (YOUNG, 1995; KLASEN, 2000).

${ }^{31}$ Como expresión fundamental de una división sexual del trabajo, las formas más desvalorizadas de trabajo son realizadas mayoritariamente por mujeres (MOLYNEUX, 1994; DE BARBIERI, 1989; RESKIN et al., 1990). Anker afirmó que la segregación ocupacional por sexo, está ampliamente extendida en todas las regiones del mundo - luego de realizar un análisis en 41 países - (ANKER, 1998). A nivel sectorial, utilizando información de Argentina, Brasil, Chile y México se detectó que gran parte de mujeres se empleaba en el sector terciario, especialmente en los sectores de salud y educación, y en el servicio doméstico; en el comercio; en la agricultura (sobre todo comercial); y en la industria tradicional como la alimenticia y la de confección de ropa (CRUZ, 2003; AQUINO, 1995).

${ }^{32}$ El creciente nivel educativo de las mujeres trabajadoras, dio cuenta a principios del nuevo siglo en la región que superaba al de los varones (CEPAL, 2001). Se destaca la concentración de mujeres en actividades dentro de las industrias de exportación; en el sector informal, sea en cadenas de subcontratación vinculadas directamente con procesos de producción industrial modernos, la agricultura comercial de exportación, o en actividades por cuenta propia relacionadas con estrategias de supervivencia doméstica (BENERÍA, 2001; CHARMES, 2000).

${ }^{33}$ La serie PBI real per cápita tiene como año base el 2011, fue extraída de la base de datos del proyecto Maddison versión 2018 (https://www.rug.nl/ggdc/historicaldevelopment/maddison/research)

${ }^{34}$ Proponen analizar los datos de crecimiento de sectores específicos como un mejor proxy para medir el impacto de la participación de las mujeres en la fuerza laboral en los países en diferentes etapas del proceso de desarrollo (GADDIS et al., 2013). 
particularmente en las mujeres en edad reproductiva y con hijos (ARIZA et al., 2002; CHANT et al., 2003; GARCÍA, 2003), en el marco de recurrentes crisis económicas que motivaron la integración de la mujer a los mercados de trabajo (GÓMEZ GÓMEZ, 1993). Este incremento de actividad económica de las mujeres se vincula también con factores demográficos y socioculturales como el descenso de la fecundidad ${ }^{35}$; transformaciones en la estructura de los hogares, y cambios culturales y simbólicos que afectan los roles tradicionales masculinos y femeninos dentro y fuera del hogar (ARIZA et al., 2001, 2001, 2003; BENERÍA, 2001; GARCÍA, 1998, 2003, 2001; GÓMEZ, 2001; GUZMÁN et al., 2001; LERNER et al., 1994; OLIVEIRA et al., 2000; OLIVEIRA, 1993; YÁÑEZ, 1999). Si bien en la región se produjo un descenso en la brecha salarial de género, persiste la segregación ocupacional. Las mujeres continúan integrándose a un número acotado de ocupaciones donde las remuneraciones percibidas siguen siendo comparativamente más bajas que en el resto del ámbito laboral (MAUBRIGADES, 2017).

De la Tabla 2, se destaca el predominio de la heterogeneidad en la brecha de género, mercados de trabajo donde la población nativa presenta las menores brecha de género -como por ejemplo Uruguay, Bolivia y Brasil -, poseen entre su población económica extranjera niveles de brecha de género marcadamente elevados -brasileños (29,1 p.p.), paraguayos (36,7 p.p.), y mexicanos (34,1 p.p.), para los mercados nacionales mencionados, respectivamente-.

Los resultados presentados abren un espacio de análisis en las desigualdades oportunidades económicas para varones y mujeres según su país de nacimiento y el destino posible de inserción laboral dentro de la región, puesto que no existen leyes de hierro que regulen la participación de las mujeres en el mercado de trabajo.

\section{BIBLIOGRAFÍA}

ACKER, J. (1990), "Hierarchies, Jobs, Bodies: A Theory of Gendered Organizations", Gender and Society, vol. 4, pp. 139-168.

ANKER, R. (1987), "Participación laboral de la mujer en los países en desarrollo: examen crítico de las definiciones y los métodos de compilación de datos", Medición de las actividades económicas de la mujer, Richard Anker y Catherine Hein (comps.), Ginebra, Organización Internacional del Trabajo (OIT).

ANKER, R. (1998), Gender and Jobs: Sex Segregation of Occupations in the World, Ginebra, International Labour Office.

AQUINO, E. (1995), "Mulher, Saúde e Trabalho no Brasil: Desafios para um Novo Agir", Cadernos de Saúde Pública, vol. 11, no. 2, pp. 281-290.

ARIZA, M. y DE OLIVEIRA, O. (2001), "Familias en transición y marcos conceptuales en redefinición", Papeles de población, vol. 28.

ARIZA, M. y DE OLIVEIRA, O. (2002), "Cambios y continuidades en el trabajo, la familia y la condición de las mujeres", en Elena Urrutia (coord.), Estudio sobre las mujeres y las relaciones de género en México: aportes desde diversas disciplinas, México, El Colegio de México, Programa Interdisciplinario de Estudios de la Mujer, pp. 43-86.

ARIZA, M. y DE OLIVEIRA, O. (2003), "Acerca de las familias y los hogares: estructura y dinámica”, en Catalina Wainerman (comp.), Familia, trabajo y género: un mundo de nuevas relaciones, Buenos Aires, Fondo de Cultura Económica, Fondo de las Naciones Unidas para la Infancia (UNICEF), pp. 19-54.

AGUILAR, M., y EPSTEIN, E. (2005). La cédula censal entre productores y demandantes de información. Trabajo presentado en III Jornadas de Jóvenes Investigado--res, Buenos Aires: $I I G G$.

ARUJ, R. S. (2008). Causas, consecuencias, efectos e impacto de las migraciones en Latinoamérica. Papeles de población, 14(55), 95-116.

\footnotetext{
${ }^{35}$ El número de hijos por mujer ha disminuido en gran parte del mundo, tanto en países desarrollados, como en países en vía de desarrollo, pasando de un promedio de 5 hijos por mujer a nivel mundial en 1950 a un promedio de 2.5 hijos por mujer en 2010 (DEL BOCA et al., 2003; SCHULTZ, 2005; NGUYEN THI HONG, 2009).
} 
BENERÍA, L. y ROLDÁN, M. (1987), The Crossroads of Class and Gender: Industrial Homework, Subcontracting and Household Dynamics in Mexico City, Chicago, University of Chicago Press.

BENERÍA, L. (1982), "Accounting for women's work", Women and Development. The Sexual Division of Labor in Rural Societies, Lourdes Benería (comp.), Nueva York, Praeger.

BENERÍA, L. (1991), "The measurement of women's economic activities: Assesing the theoretical and practical work of two decades", documento presentado al Meeting of Experts on Social Development Indicators, Rabat, Marruecos, 8 al 11 de abril.

BENERÍA, L. (1992), “Accounting for Women's Work: Assessing the Progress of two Decades",World Development, vol. 20, no. 11, pp. 1547-1560.

BENERÍA, L. (2001), "Changing Employment Patterns and the Informalization of Jobs: General Trends and Gender Dimensions", Geneva, International Labour Office, [En línea], Disponible en: http://www.ilo.org/public/english/protection/ses/download/docs/gender.pdf

BLADES, D.W. (1975), "Non-monetary (subsistence) activities in the national accounts of developing countries", París, Organización de Cooperación y Desarollo Económicos (OCDE).

BOHOSLAVSKY, E. (2009). ¿ Qué es América Latina? El nombre, la cosa y las complicaciones para hablar de ellos. Ponencia. Taller de reflexión sobre América Latina, Universidad Nacional de General Sarmiento, Los Polvorines, 15, 1-12.

BOSERUP, E. (1970). Women's role in economic development. Nueva York, St. Martin's Press.

BUVINIC, M., GIUFFRIDA, A. y GLASSMAN, A. (2002), Gender Inequality in Health and Work: the Case for Latin America and the Caribbean, Washington D. C., Sustainable Development Department, Technical Papers Series No. SOC-128, Inter-American Development Bank.

CAMOU, M. M. y MAUBRIGADES, S. (2011). Sesgo de género en el crecimiento económico: comparaciones latinoamericanas. 5tas Jornadas de Historia Económica, Montevideo.

CELADE (1991). Haití: Caracterización demográfica y su impacto sobre los servicios sociales. Santiago, Chile. Disponible en https://repositorio.cepal.org/bitstream/handle/11362/32689/D16777.00_es.pdf;jsessionid=294F08E502B917E87580156CDC1950EB?sequence $=1$

CEPAL (Comisión Económica para AmÉRICA LATINA Y EL CARIBE) (1995), Programa de Acción Regional para las Mujeres de América Latina y el Caribe, 1995-2001 (LC/G.1855), Santiago de Chile.

CHANT, S. y CRASKE, N. (2003), Gender in Latin America, Nuevo Brunswick, Nueva Jersey, Rutgers University Press.

CHARMES, J. (2000), "Informal Sector, Poverty and Gender: A Review of Empirical Evidence", Background Paper for the World Development Report, 2001.

CRUZ, A., NORIEGA, M., y GARDUÑO, M. (2003). Trabajo remunerado, trabajo doméstico y salud: las diferencias cualitativas y cuantitativas entre mujeres y varones. Cadernos de Saúde Pública, 19, 1129-1138.

COLLIER, D. (1993). El método comparativo: dos décadas de cambios. La comparación en las Ciencias Sociales, Alianza Editorial.

COURTIS, C. y PACECCA, M. (2010). "Género y trayectoria migratoria: mujeres migrantes y trabajo doméstico en el Área Metropolitana de Buenos Aires", Papeles de Población, vol. 16, núm. 63. pp. 155-185.

DAEREN, L. (2001). Enfoque de género en la política económica-laboral. El Estado de arte en América Latina y el Caribe, en: Serie Mujer y Desarrollo, $\mathrm{n}^{\circ}$ 29, Santiago de Chile, CEPAL.

DE BARBIERI, M. (1989), "Trabajos de la reproducción”, en Orlandina de Oliveira, Marielle Pepin Lehauller y Vania Salles (comps.), Grupos domésticos y reproducción cotidiana, México, Porrúa, pp.235-254.

DE BRITO, J. (1997), "Uma proposta de vigilância en saúde do trabalhador com a ótica de gênero", Cadernos de Saude Pública, vol. 13 (suplemento 2), pp. 141-144

DEL BOCA, D. y PASQUA, S. (2003), "Employment Patterns of Husbands and Wives and Family Income Distribution in Italy (1977-1998)", forthcoming in Review of Income and 
Wealth.

DEPARTAMENTO ADMINISTRATIVO NACIONAL DE ESTADÍSTICA. (s.f.). Censo Básico de Colombia 2005. Recuperado de http://systema59.dane.gov.co/cgibin/RpWebEngine.exe/PortalAction?BASE=CG2005BASI $\mathrm{CO}$

DIRECCIÓN DE ESTADÍSTICA Y CENSO. (s.f.). Boleta Censal Panamá 2010. Recuperado de http://estadisticas.contraloria.gob.pa/inec/cgi bin/RpWebEngine.exe/Portal?\&BASE=LP2010\&MAIN=WebServerMain_censos.inlenso05 .pdf

DIRECCIÓN GENERAL DE ESTADÍSTICA Y CENSOS. (s.f.). Boleta Censal El Salvador 2007. Recuperado de http://www.digestyc.gob.sv/servers/redatam/htdocs/CPV2007S/Docs/BOLETA.pdf

DIRECCIÓN GENERAL DE ESTADÍSTICA, ENCUESTAS Y CENSOS. (s.f.). Boleta Censal Paraguay 2012. Recuperado de https://celade.cepal.org/censosinfo/Boletas/PY_BDef_2012.pdf

DIXON-MUELLER, R. y ANKER, R. (1988), "Assessing women's economic contribution to development", Training in Population, Human Resources and Development Planning, $\mathrm{N}^{\mathrm{o}}$ 6, Ginebra, Organización Internacional del Trabajo (OIT)

DOLLAR, D. y GATTI, R. (1999). Gender Inequality, Income, and Growth: Are Good Times Good for Women? Policy research report on gender and development. Working Paper Series, No. 1, The World Bank.

ELIZAGA, J. y MELLON, R. (1971). Aspectos demográficos de la mano de obra en América Latina. Santiago de Chile: CELADE.

FAURA MARTÍNEZ, Ú. y GÓMEZ GARCÍA, J. (2002). ¿Cómo medir los flujos migratorios? Papers, 66, 15-44. Universidad de Murcia.

FORSYTHE, N., KORZENIEWICZ, R., y DURRANT, V. (2000). Gender Inequalities and Economic Growth: A Longitudinal Evaluation. Economic Development and Cultural Change, 48, 573-617.

GADDIS, I. y KLASEN, S. (2013). "Economic development, structural change, and women's labor force participation." Journal of Population Economics: 1-43.

GADDIS, I. y KLASEN, S. (2014). "Economic development, structural change, and women's labor force participation." Journal of Population Economics 27(3): 639-681.

GOLDIN, C. (2006). "The Quiet Revolution That Transformed Women's Employment, Education, and Family." The American Economic Review 96(2): 1-21

GARCÍA, B. (1998), "Comentarios al trabajo "Investigación sobre trabajo femenino y salud: avances y propuestas", en J. G. Figueroa (comp.), La condición de la mujer en el espacio de la salud, México, El Colegio de México, pp. 161-164.

GARCÍA, B. (2001), "Dinámica familiar y calidad de vida", en Beatriz Figueroa Campos (coord.), México diverso y desigual: enfoques sociodemográficos. V Reunión de investigación sociodemográfica en México, vol. 4, México, El Colegio de México, Centro de Estudios Demográficos y de Desarrollo Urbano, pp. 129-175.

GARCÍA, B. (2003), "Reestructuración económica, trabajo y autonomía femenina en México", en Elena Urrutia (coord.), Estudio sobre las mujeres y las relaciones de género en México: aportes desde diversas disciplinas, México, El Colegio de México, Programa Interdisciplinario de Estudios de la Mujer, pp. 87-120.

GARCÍA, B. y DE OLIVEIRA, O. (1994), Trabajo femenino y vida familiar en México, México, El Colegio de México.

GÓMEZ GÓMEZ, E. (1993), "Introduction", Gender, Women and Health in the Americas, Washington, Pan American Health Organization, World Health Organization, pp. IX-XIX.

GÓMEZ, N. (2001), "Mujeres, manufactura y liberalización económica, 1990-1997: ¿qué tanto hemos ganado?", en Jennifer A. Cooper (coord.), ¿Esto es cosa de hombres? Trabajo, Género y Cambio Social, México, UNAM, Coordinación de Humanidades, Programa Universitario de Estudios de Género, pp. 203-224.

GRIMOLDI, N. (2014) • ¿De qué hablamos cuando hablamos de América Latina? Un análisis a 
través de las imágenes: La identidad de la región en los lenguajes visuales y las imágenes que circulan en medios de comunicación y en la vía pública, La Plata 2008-2013. Trabajo final de grado. Universidad Nacional de La Plata. Facultad de Humanidades y Ciencias de la Educación. En Memoria Académica.

GUZMÁN, V. y TODARO, R. (2001), “Apuntes sobre el género en la economía global”, El género en la economía, no. 32, Santiago de Chile, Centro de Estudios de la mujer, ISIS Internacional

HARLOW, S., DENMAN, C., y CEDILlO, L. (2004), "Occupational and Population Health Profiles: A Public Health Perspective on the Social Costs and Benefits of Export-led Development", en Kathy Kopinak (ed.), Social Costs of Industrial and Urban Growth in Northern Mexico, La Jolla, CA, Center for US-Mexican Studies, University of California, San Diego.

HILFINGER, M., DEANNE, K., EUN-OK IM, PAGE, A., REGEV, H., SPIERS, J., YODER L., AFAF, I. (1997), "Defining and Redefining Work: Implications for Women's Health", Gender and Society, vol. 11, no. 3, pp. 296-323.

INSTITUTO BRASILEÑO DE GEOGRAFÍA Y ESTADÍSTICA (IBGE). (s.f.). [Cuestionario Básico. Censo Demográfico 2010]. Recuperado de https://biblioteca.ibge.gov.br/visualizacao/instrumentos_de_coleta/doc3128.pdf

INSTITUTO NACIONAL DE ESTADISTICAS Y CENSOS (INDEC). (s.f.). [Cuestionario básico de viviendas particulares]. Recuperado de https://www.indec.gob.ar/ftp/cuadros/poblacion/cuestionario_basico_2010.pdf

INSTITUTO NACIONAL DE ESTADÍSTICA (INE). (s.f.-a). Boleta Censal Uuruguay 2011.

Recuperado

https://www.redatam.org/redury/censos/cpv2011/Cuestionario_VIV_HOG_PERS.pdf

INSTITUTO NACIONAL DE ESTADÍSTICA (INE). (s.f.-b). Cuestionario del Empadronamiento General del Censo de Venezuela20112011. Recuperado de http://www.ine.gov.ve/documentos/Demografia/CensodePoblacionyVivienda/pdf/Cuestionar io_Censo_2011.pdf

INSTITUTO NACIONAL DE ESTADÍSTICA DE BOLIVIA (INE). (s.f.). [Boleta censal de $\begin{array}{llll}\text { Población } & \text { y } & \text { 2012]. } & \text { Rivienda de }\end{array}$ http://datos.ine.gob.bo/redbol/censos/cpv2012/BoletaCensal2012.pdf

INSTITUTO NACIONAL DE ESTADÍSTICA DE GUATEMALA. (s.f.). Boleta Censal $\begin{array}{llll}\text { Particulares } & \text { Guatemala } & \text { 2018. } & \text { Recuperado de }\end{array}$ http://redatam.censopoblacion.gt/redgtm/censos/cen2018/docs/GT_BDef_2018.pdf

INSTITUTO NACIONAL DE ESTADÍSTICA E INFORMÁTICA. (s.f.). Cedula Censal Perú 2007. Recuperado de http://censos.inei.gob.pe/Censos2007/redatam/doc/CedulaCensal.pdf

INSTITUTO NACIONAL DE ESTADÍSTICA Y CENSOS (INEC). (s.f.). Boleta Censal Nicaragua $2005 . \quad$ Recuperado de https://www.inide.gob.ni/censos2005/bolfeb22/BolCenso05.pdf

INSTITUTO NACIONAL DE ESTADÍSTICA Y CENSOS DE COSTA RICA (INEC). (s.f.). Boleta Censal de Costa Rica 2011. Recuperado de http://sistemas.inec.cr:8080/bincri/RpWebEngine.exe/Portal?BASE=2011

INSTITUTO NACIONAL DE ESTADÍSTICA Y CENSOS DEL ECUADOR (INEC). (s.f.). Cuestionario Censal de Ecuador 2010. Recuperado de http://redatam.inec.gob.ec/cgibin/RpWebEngine.exe/PortalAction?\&MODE=MAIN\&BASE $=$ CPV2010\&MAIN $=$ WebServerMain.inl

INSTITUTO NACIONAL DE ESTADÍSTICA Y GEOGRAFÍA (INEGI). (s.f.). Cuestionario Básico México 2010. Recuperado de https://www.inegi.org.mx/contenidos/programas/ccpv/2010/doc/cpv2010_cuest_basico_d.pd f

INSTITUTO NACIONAL DE ESTADÍSTICAS DE CHILE (INE). (s.f.). [Cuestionario Censal Censo 2002]. Recuperado de http://redatam-ine.ine.cl//cuestionarios/C2002.pdf

INSTITUTO NACIONAL DE ESTADÍSTICAS HONDURAS (INE). (s.f.). Cuestionario Censal Honduras 2013.2 Recuperado de http://170.238.108.227/redhnd/censos/cpv2013/Formulario_CNPV_2013.PDF 
IZQUIERDO, M. (1998), Aguantando el tipo. Desigualdad social y discriminación salarial, Barcelona, Diputació de Barcelona.

KERGOAT, D. (2003), "De la relación social de sexo al sujeto sexuado", Revista Mexicana de Sociología, año 65, no. 4, pp. 841-861.

KLASEN, S. (2000): "Does gender inequality reduce growth and development? Evidence from cross-country regressions", World Bank Policy Research Report on Gender and Development, Working Paper Series, $\mathrm{N}^{\circ} 7$.

LARA, M. (1998), "Investigación sobre trabajo femenino y salud: avances y propuestas", en G. Figueroa (comp.), La condición de la mujer en el espacio de la salud, México, El Colegio de México, pp. 131-160.

LASLETT, B. y BRENNER, J. (1989), "Gender and Social Reproduction: Historical Perspectives", Annual Review of Sociology, no. 15, pp. 381-404.

LERNER, Susana, QUESNEL, A. y YÁÑES, M. (1994), "La pluralidad de trayectorias reproductivas y las transacciones institucionales", Estudios Demográficos y Urbanos. 27, vol. 9, no. 3, pp. 543- 578.

LIPSZYC, C. (2004). Feminización de las migraciones: sueños y realidades de las mujeres migrantes en cuatro países de América Latina. Seminario "Caminar sin Miedos", UrbalRed, 12.

LUTZEL, H. (1989), "Household production and national accounts", documento presentado a la Segunda Reunión Conjunta ECE/INSTRAW, Ginebra, 13-16 de noviembre.

MADDISON PROJECT DATABASE, (2018). Bolt, Jutta, Robert Inklaar, Herman de Jong y Jan Luiten van Zanden (2018), " Rebasar 'Maddison': nuevas comparaciones de ingresos y la forma del desarrollo económico a largo plazo", recuperado de: https://www.rug.nl/ggdc/historicaldevelopment/maddison/releases/maddison-projectdatabase-2018

MANZANO, F., y VELÁZQUEZ, G. (2016). El rol del espacio geográfico como determinante de la participación laboral. Argentina (2001-2010). Revista Tamoios, 12(1).

MÁRQUEZ, M.; TALAMANTE, C. y GARDUÑO, M. A., (1993). La Salud Enfermedad de las Maestras: una Experiencia Participativa. Ponencia presentada en el Coloquio: Género y Salud Femenina: Enfoques y Perspectivas, 23-25 Junio 1993, México, D.F.: CIESAS-INNSZINESER

MARTÍNEZ PIZARRO, J.; CANO CHRISTINY, V.; SOFFIA CONTRUCCI, M. (2014). Tendencias y patrones de la migración latinoamericana y caribeña hacia 2010 y desafíos para una agenda regional. Serie Población y Desarrollo. CEPAL

MARTÍNEZ, J. y VONO, D. (2005). Geografía migratoria intrarregional de América Latina y el Caribe al comienzo del siglo XXI. Revista de Geografía Norte Grande, n.34.

MARTÍNEZ PIZARRO, J. (2003). El mapa migratorio de América Latina y el Caribe, las mujeres y el género, serie Población y Desarrollo, No 44 (LC/L.1974-P), Santiago, CEPAL.

MAUBRIGADES, S. (2017). Las mujeres en el mercado de trabajo en América Latina durante el siglo XX: un análisis comparado de la tasa de actividad, sus factores explicativos y su impacto en la brecha salarial.

MIKKOLA, A. (2005). "Role of Gender Equality in Development - A Literature Review." SSRN Electronic Journal. http://www.ssrn.com/abstract=871461

MOLYNEUX, M. (1994), "Más allá del debate sobre el trabajo doméstico", en Cristina Borderías, Cristina Carrasco y Carmen Alemany (comps.), Las mujeres y el trabajo. Rupturas conceptuales, Barcelona, Icaria, pp. 111-150

MUÑOZ, O. (2009). Factores determinantes de la participación laboral: aspectos conceptuales. Tendencias, Colombia, v.10, n. 1, p. 87-116.

NGUYEN THI HONG, T. (2009). "The effects of fertility on female labor supply."

OFICINA NACIONAL DE ESTADÍSTICA. (s.f.). Cédula Censal República Dominicana 2010. Recuperado de http://redatam.one.gob.do/redatam/do/CPV2010/Docs/Boleta_Censal.pdf

OFICINA NACIONAL DE ESTADÍSTICAS DE CUBA (ONE). (s.f.). Cuestionario Censal de Cuba 2012. Recuperado de http://www.one.cu/publicaciones/cepde/cpv2012/documentacion_censal/Modelo\%20C1.\%2 0Cuestionario\%20Censal.pdf 
OLIVEIRA, M. (1993), "Women on their Own: Alternatives of Domestic Arrangements at Different Stages of the Life Course", trabajo presentado en el XXII Congreso General de Población. Unión Internacional para el Estudio Científico de la Población, Montreal, Canadá, 24 de agosto-01 de septiembre.

OLIVEIRA, O. y ARIZA, M. (2000), "Género, trabajo y exclusión social en México", Estudios Demográficos y Urbanos, vol. 15, no. 1, pp. 11-33.

OLIVEIRA, O., ETERNOD, M. y LÓPEZ, M. (2000), "Familia y género en el análisis sociodemográfico", en Brígida García (coord.), Mujer, género y población en México”, México, El Colegio de México, Sociedad Mexicana de Demografía, pp. 211-271. Ortiz, Sutti (2002), "Laboring in the Factories and in the Fields", Annual Review of Anthropology, vol.31, pp. 395-417.

PAMPEL, F. y TANAKA, K. (1986). "Economic Development and Female Labor Force Participation: A Reconsideration." Social Forces 64(3): 599-619.

PANIGO, D., PÉREZ, P., y PERSIA, J. (2014). Actividad, empleo y desempleo. Buenos Aires: CEIL CONICET. Trabajo y Sociedad.

PEDRERO, M. (2004), "Género, trabajo doméstico y extradoméstico en México. Una estimación del valor económico del trabajo doméstico", Estudios demográficos y urbanos, vol. 19, no. 2 (56), pp. 413-446.

POLLACK, E. M. (1997). "Reflexiones sobre los indicadores del mercado de trabajo para el diseño de políticas con un enfoque basado en el género."

PSACHAROPOULOS, G. y TZANNATOS, Z. (1989). "Female Labor Force Participation: An International Perspective." World Bank Research Observer 4(2): 187-201.

RAVELO BLANCAS, P. (1995), "Perspectivas teórico-metodológicas para el estudio de la salud en el trabajo femenino", en Sara Elena Pérez-Gil Romo, et al. (coords.), Género y Salud Femenina, México, CIESAS, Universidad de Guadalajara, Instituto de Nutrición Salvador Zubirán, pp. 217-241.

RESERVE, R. (2014). Haití: ¿ la misión de la última oportunidad?. Revista de ciencia política (Santiago), 34(1), 189-201.

RESKIN, Barbara y ROOS, P. (1990), Job Queues, Gender Queues, Filadelfia, Temple University Press.

SCHULTZ, T. (2005). "Fertility and income." Yale University Economic Growth Center Discussion Paper (925).

SEGUINO, S. (2000). "Accounting for Gender in Asian Economic Growth." Feminist Economics 6(3): 27-58

SHAW, R. (1975). Migration Theory and Fact: Review and Bibliography of Current Literature. Series Bibliográficas, 5. Philadelphia: Regional Science Research Institute.

STALKER, P. (1994). "Trabajando juntos", cap. ¿Porqué migra la gente?, en The work of stranger, OIT, Ginebra.

SZASZ, I. (1999), "Género y salud. Propuestas para el análisis de una relación compleja", en M. N. Bronfman y R. Castro (coords.), Salud, cambio social y política: perspectivas desde América Latina, México, Instituto Nacional de Salud Pública, Foro Internacional de Ciencias Sociales y Salud, Edamex, pp. 109- 121.

TZANNATOS, Z. (1999). "Women and Labor Market Changes in the Global Economy: Growth Helps, Inequalities Hurt and Public Policy Matters." World Development 27(3): 551-569.

VANDELAC, L. (1994). "La economía doméstica a la salsa mercantil... o las valoraciones monetarias del trabajo doméstico", en Cristina Borderías, Cristina Carrasco y Carmen Alemany (comps.), Las mujeres y el trabajo. Rupturas conceptuales, Barcelona, Icaria, pp.151-208.

VILLA, M. y MARTÍNEZ, J. (2002). Rasgos sociodemográficos y económicos de la migración internacional en América Latina y el Caribe. Capítulos, n. 65, p. 26-67.

WAINERMAN, Catalina H. y RECCHINI DE LATTES, Z. (1981), "El trabajo femenino en el banquillo de los acusados", Population Council/Terra Nova México, D.F.

YÁÑEZ, S. (1999), "Consideraciones sobre flexibilidad laboral planteadas desde una mirada de 
género", en Ensignia, J. y S. Yáñez (eds.), Sindicalismo, género y flexibilización en el MERCOSUR y Chile: inserción laboral femenina, Santiago de Chile, Fundación Friedrich Ebert (representación Chile) y Centro de Estudios de la Mujer, pp. 97-110.

YOUNG, A. (1995): "The Tyranny of Numbers: Confronting the Statistical Realities of the East Asian Growth Experience." The Quarterly Journal of Economics 110 (3, August): 641-680

\section{ANEXO}

TABLA 3

Tasa de actividad varones según país de nacimiento. Países de América Latina en fechas alrededor del año 2010.

\begin{tabular}{|c|c|c|c|c|c|c|c|c|c|c|c|c|c|c|c|c|c|c|}
\hline 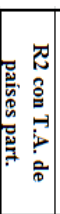 & $?$ & 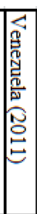 & & 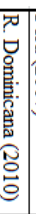 & & & & & 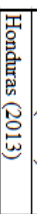 & & & & 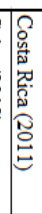 & & & & 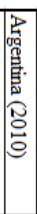 & 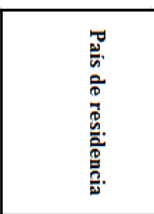 \\
\hline & & 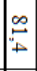 & 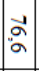 & $\begin{array}{c}\infty \\
\infty \\
\infty \\
\infty\end{array}$ & & & 望 & \begin{tabular}{l}
$\infty$ \\
\hdashline \\
\hdashline \\
\hdashline
\end{tabular} & बे & 党. & 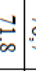 & 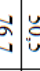 & $\infty$ & 足 & & 学 & 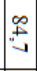 & Latinoamérica \\
\hline 兽 & $\underset{\omega}{\vec{w}}$ & 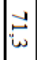 & 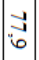 & $\begin{array}{c}-1 \\
0 \\
\text { in }\end{array}$ & & 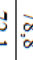 & & $\mid$\begin{tabular}{l}
$\infty$ \\
\hdashline \\
\hdashline
\end{tabular} & ă & $\begin{array}{c}\sigma \\
\infty \\
\infty\end{array}$ & 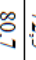 & W & I & \begin{tabular}{l}
$a$ \\
\hdashline \\
$+\infty$
\end{tabular} & g) & $\stackrel{t}{t}$ & $\because$ & Argentina \\
\hline ్ㅗ & $\underset{\sim}{\vec{\omega}}$ & 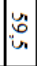 & 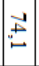 & : & & $:$ & & $\begin{array}{l}\infty \\
\infty \\
\vdots\end{array}$ & & & 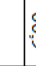 & 8 & : & . & & : & 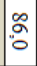 & Bolivia \\
\hline$\stackrel{\circ}{\breve{~}}$ & ఫே & 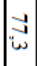 & $\vec{\omega}$ & $\begin{array}{l}\text {. } \\
\text { :ै }\end{array}$ & & 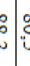 & & 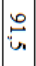 & 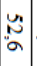 & & $\begin{array}{l}a \\
\infty \\
+ \\
+\end{array}$ & . & Iิ & . & & is & N & Brasil \\
\hline$\underset{亡}{\stackrel{0}{\infty}}$ & है & 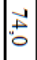 & $\ddot{0}$ & $\begin{array}{l}-1 \\
\infty \\
\infty \\
\text {. }\end{array}$ & : & 0 & & 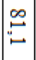 & $\mid \begin{array}{l}: \\
\ddot{0}\end{array}$ & & 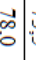 & 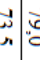 & on & वे. & 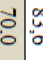 & is & 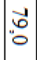 & Chile \\
\hline${\underset{\infty}{\infty}}_{\infty}^{O}$ & $\ddot{\circ}$ & $\stackrel{\infty}{\circ}$ & $\because$ & $\begin{array}{l}-\overrightarrow{0} \\
\stackrel{\infty}{+}\end{array}$ & & 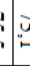 & & 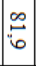 & 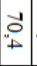 & $\begin{array}{l}1 \\
.6 \\
+\end{array}$ & $\begin{array}{cc}-1 \\
0\end{array}$ & u. & $\because$ & 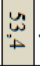 & $\begin{array}{lll}0 & 0 \\
0\end{array}$ & 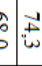 & $\stackrel{\infty}{=}$ & Colombia \\
\hline 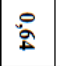 & $\vec{\square}$ & $\$$ & & 。ั & & 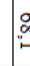 & $\frac{\mathrm{u}}{\circ}$ & \begin{tabular}{l}
0 \\
\hdashline \\
\hdashline \\
\hdashline
\end{tabular} & 早 & $\stackrel{5}{5}$ & $\begin{array}{l}0 \\
\vdots \\
\vdots\end{array}$ & $\begin{array}{c}a \\
\infty \\
\infty\end{array}$ & $\therefore$ & 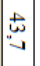 & & & $\begin{array}{l}\vec{w} \\
\oplus \\
+\end{array}$ & Costa Rica \\
\hline 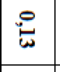 & $\stackrel{\infty}{a}$ & $\begin{array}{l}\infty \\
\cdots \\
\alpha\end{array}$ & $\begin{array}{l}\infty \\
\vdots \\
\vdots\end{array}$ & $\begin{array}{c}7 \\
\infty \\
\infty\end{array}$ & & . & is & $\begin{array}{l}\infty \\
\vdots \\
0 \\
\end{array}$ & $\vec{\sim}$ & $\begin{array}{l}\infty \\
\infty \\
\infty\end{array}$ & : & 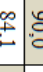 & 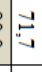 & $\overrightarrow{.1}$ & $\stackrel{i}{\infty}$ & $\begin{array}{l}0 \\
0\end{array}$ & is & Cuba \\
\hline 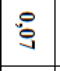 & : & 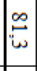 & $:$ & $\begin{array}{l}-1 \\
0 \\
0 \\
0\end{array}$ & & 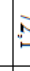 & & $\begin{array}{c}\infty \\
\infty \\
\infty \\
\infty\end{array}$ & & & هُ & o. & वे & $\begin{array}{l}8 \\
+ \\
+ \\
\end{array}$ & $\vec{\infty}$ & 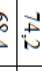 & के & Ecuador \\
\hline 옴 & $\Xi$ & $\begin{array}{l}\overrightarrow{2} \\
- \\
\end{array}$ & & 㕝 & & 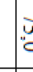 & $\underset{\infty}{+\infty}$ & $\begin{array}{l}\infty \\
0 \\
0\end{array}$ & . & & $\begin{array}{c}u \\
\infty \\
\omega \\
\omega\end{array}$ & & जै & $\mid \begin{array}{l}a \\
\vec{a} \\
\vdots\end{array}$ & & & 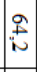 & El Salvador \\
\hline 芯 & $\underset{\omega}{\vec{u}}$ & & & 志 & & 8 & سّ & 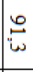 & $\stackrel{\leftrightarrow}{\sim}$ & & $\begin{array}{l}\infty \\
\dot{a} \\
i n\end{array}$ & & $\begin{array}{l}\infty \\
\infty \\
\infty\end{array}$ & 竞 & & & $\begin{array}{l}3 \\
\infty\end{array}$ & Guatemala \\
\hline 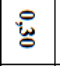 & 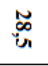 & 岃 & & $\begin{array}{lll}\infty \\
: 0 \\
0\end{array}$ & & is & & & & & & ט. & $\vec{\Delta}$ & & & & Iี & Haití \\
\hline 品 & $\stackrel{t}{6}$ & & & à & & + & & ים & $\begin{array}{l}u \\
u \\
w \\
w \\
w\end{array}$ & 。े & $\begin{array}{c}a \\
\infty \\
\infty \\
0\end{array}$ & & \begin{tabular}{l}
$\infty$ \\
\hdashline \\
+
\end{tabular} & $\begin{array}{l} \\
u \\
a \\
a\end{array}$ & & & जa & Honduras \\
\hline $\begin{array}{l}0 \\
0 \\
0\end{array}$ & $\because$ & ने & $\infty$ & $\begin{array}{l}\text { so } \\
\text { is } \\
\end{array}$ & 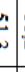 & sing & ò & 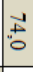 & $\begin{array}{l}\text { un } \\
\text { o. } \\
\infty\end{array}$ & $\begin{array}{l}\overrightarrow{3} \\
+ \\
+\end{array}$ & + & $\begin{array}{ll}0 \\
0\end{array}$ & $\frac{1}{\infty}$ & 茨 & 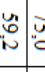 & & ت: & México \\
\hline$\stackrel{\circ}{\circ}$ & $\vec{g}$ & o & & : & & 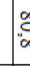 & is & $\begin{array}{l}\infty \\
0 \\
0 \\
0\end{array}$ & $\begin{array}{l}\vec{z} \\
\text { un }\end{array}$ & . & : & $\exists$ & $\stackrel{\infty}{\infty}$ & 产 & & & $\stackrel{\infty}{\sim}$ & Nicaragua \\
\hline$\underset{\mathrm{U}}{0}$ & $\begin{array}{l}\vec{b} \\
\text { in }\end{array}$ & 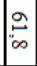 & & 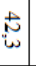 & & ‡ & & 总 & 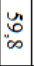 & o. & : & & $\therefore$ & 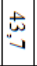 & $+\infty$ & & 文 & Panamá \\
\hline 品 & $\underset{\infty}{n}$ & & $\begin{array}{l}2 \\
+ \\
\end{array}$ & & & & & \begin{tabular}{l}
0 \\
\hdashline \\
\hdashline
\end{tabular} & & & & & & \begin{tabular}{|l|} 
\\
\\
$\infty$ \\
\end{tabular} & $\begin{array}{cc}+ \\
\infty \\
0\end{array}$ & 党 & $\begin{array}{l}\infty \\
\pm \\
0 \\
0\end{array}$ & Paraguay \\
\hline : & $\ddot{b}$ & {$\left[\begin{array}{l}\infty \\
\omega \\
\cdots\end{array}\right.$} & \begin{tabular}{l}
$\infty$ \\
\hdashline \\
$\sim$ \\
$\sim$
\end{tabular} & $\begin{array}{c}\infty \\
\vdots \\
\vdots\end{array}$ & s & $t^{\circ}$ & & : & $\stackrel{\square}{\longleftarrow}$ & ه' & 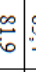 & \begin{tabular}{l}
$\infty$ \\
\hdashline \\
\hdashline \\
\end{tabular} & $\stackrel{\infty}{\mathscr{u}}$ & $\begin{array}{l}\vec{\omega} \\
\vec{\omega} \\
\vec{*}\end{array}$ & \begin{tabular}{c}
$\infty$ \\
\hdashline \\
$-\infty$
\end{tabular} & $\begin{array}{ll}4 \\
\Delta\end{array}$ & 象 & Perú \\
\hline$\stackrel{\circ}{\infty}$ & $\underset{+}{\mathbb{N}}$ & \begin{tabular}{|c}
$\infty$ \\
0 \\
$\infty$ \\
$\infty$
\end{tabular} & & जे & & sis & & is & $\begin{array}{l}8 \\
+ \\
+ \\
\end{array}$ & & & & חי & $\mid \begin{array}{l}u \\
0 \\
w \\
\omega\end{array}$ & & & $\begin{array}{l}\infty \\
+ \\
\infty \\
\infty\end{array}$ & R. Dominicana \\
\hline : & ă & $\vec{a}$ & $\begin{array}{l}0 \\
\infty \\
\infty\end{array}$ & $\stackrel{\infty}{\circ}$ & & g & & $\begin{array}{l}\infty \\
\stackrel{0}{0} \\
0\end{array}$ & & & & $\begin{array}{l}6 \\
6 \\
0\end{array}$ & $\begin{array}{l}\infty \\
\text { w } \\
\text { N }\end{array}$ & $\begin{array}{ll} \\
\vdots \\
\infty \\
\infty\end{array}$ & 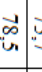 & a & $\begin{array}{l}\infty \\
\cdots \\
+\infty\end{array}$ & Uruguay \\
\hline 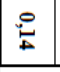 & 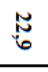 & $\overrightarrow{0}$ & 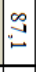 & $\begin{array}{c}\infty \\
\infty \\
-1\end{array}$ & & T & & $\begin{array}{l}\infty \\
0 \\
0 \\
0\end{array}$ & & $\begin{array}{l}8 \\
8 \\
0\end{array}$ & a. & ب. & : & \begin{tabular}{|l|l}
$\mathbf{N}$ \\
$\vdots$ \\
0
\end{tabular} & 虫 & & 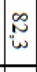 & Venezuela \\
\hline & & a & 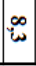 & $\circ$ & & & & $a$ & in & \begin{tabular}{c}
$\infty$ \\
\hdashline \\
$a$
\end{tabular} & $a$ & E & $\omega$ & a & a' & & 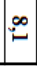 & 4 \\
\hline
\end{tabular}

Fuente: Elaboración personal en base a datos de los censos de población de los países de América Latina y del Proyecto IMILA del CELADE. 
TABLA 4

Tasa de actividad mujeres según país de nacimiento. Países de América Latina en fechas alrededor del año 2010.

\begin{tabular}{|c|c|c|c|c|c|c|c|c|c|c|c|c|c|c|c|c|c|c|c|}
\hline 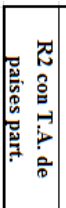 & $?$ & 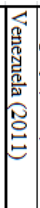 & & & & & & & & & & & & & & & & & 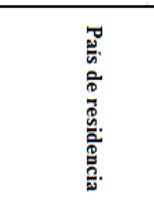 \\
\hline & & 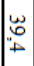 & $\begin{array}{l}4 \\
\substack{\infty \\
\infty} \\
.\end{array}$ & $\begin{array}{l}. \\
. \\
+ \\
+\end{array}$ & $\stackrel{c}{\infty}$ & & 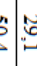 & & & & & & & & & & 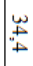 & $\stackrel{2}{-2}$ & Latinoamérica \\
\hline ¿ّ & $\underset{\substack{\mathbb{N} \\
\mathbb{N}}}{\mathbb{N}}$ & 志 & \begin{tabular}{l}
$u_{\infty}$ \\
\hdashline \\
$-\infty$
\end{tabular} & 幽. & 蓉 & $t$ & & $\vec{t}$ & & 京 & : & 古 & & 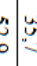 & & & 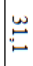 & 呆 & Argentina \\
\hline 怘 & $\underset{\infty}{\infty}$ & w & 崩. & $\begin{array}{c}\overrightarrow{.} \\
\text { is }\end{array}$ & $\begin{array}{c}w \\
\dot{u} \\
u\end{array}$ & 孞 & & un & & & & है & & 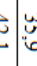 & & 8 & $\stackrel{+}{\sim}$ & $\therefore$ & Bolivia \\
\hline$\stackrel{\text { ¿ }}{\infty}$ & $\stackrel{\mathfrak{s}}{\sim}$ & $\mid \begin{array}{l}\omega \\
\infty \\
\infty \\
\sim\end{array}$ & $\begin{array}{l} \pm \\
0 \\
0\end{array}$ & $\begin{array}{c}\overrightarrow{+} \\
\infty \\
\infty\end{array}$ & 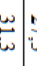 & c. & & t & 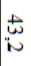 & 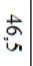 & . & $\underset{\infty}{+}$ & & u & 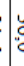 & & 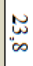 & 南 & Brasil \\
\hline 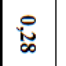 & $\stackrel{\mathfrak{s}}{\sim}$ & $\stackrel{+}{-5}$ & 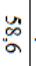 & $\begin{array}{c}\vec{y} \\
\vec{n}\end{array}$ & 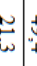 & $\begin{array}{ll}t \\
t \\
t\end{array}$ & 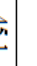 & $\underset{\infty}{\stackrel{\omega}{\infty}}$ & + & & to & $\begin{array}{l}\text { t. } \\
\text { b. }\end{array}$ & 5 & 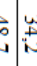 & 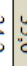 & & $\underset{\sim}{*}$ & $\stackrel{\sim}{\sim}$ & Chile \\
\hline$\stackrel{8}{ \pm}$ & $\stackrel{N}{\sim}$ & $\underset{\sim}{\mathbf{N}}$ & $\stackrel{a}{a}$ & $\stackrel{a}{=}$ & $\begin{array}{c}w \\
\dot{w} \\
\end{array}$ & 志 & 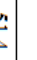 & $\ddot{\omega}$ & 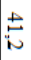 & $\begin{array}{l}\vec{t} \\
b\end{array}$ & . & 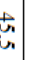 & & $\approx$ & t. & + & $\underset{0}{*}$ & $\stackrel{2}{=}$ & Colombia \\
\hline$\stackrel{i}{t}$ & $\underset{⿱ 亡}{\stackrel{\omega}{*}}$ & 岕 & & 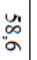 & & & 5 & $\vec{\infty}$ & . & $\begin{array}{l}+ \\
0 \\
0\end{array}$ & $\begin{array}{c}\vec{t} \\
\mathrm{~b}\end{array}$ & $\begin{array}{l}0 \\
+ \\
+ \\
+\end{array}$ & 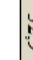 & 0 & & & & i⿱ & Costa Rica \\
\hline$\stackrel{\circ}{\Xi}$ & $\begin{array}{l}\tilde{N} \\
0\end{array}$ & '. & . & 幽. & 党 & 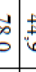 & 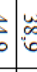 & $\underset{\text { N }}{ \pm}$ & 志 & $\begin{array}{l}9 \\
\infty \\
\infty\end{array}$ & 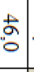 & $\begin{array}{l}2 \\
+ \\
+\end{array}$ & 家 & to & 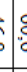 & 。 & $\infty$ & 刿 & Cuba \\
\hline$\stackrel{2}{亡}$ & $\underset{\tilde{\omega}}{\tilde{W}}$ & $\begin{array}{c}+ \\
\infty \\
\text { in }\end{array}$ & $\stackrel{a}{\dot{\omega}}$ & 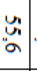 & & 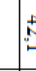 & $\overrightarrow{0}$ & 㟧 & & & $\begin{array}{c}\vec{*} \\
\dot{u}\end{array}$ & 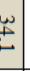 & & 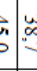 & & : & U. & के & Ecuador \\
\hline เू้ & $\begin{array}{l}b \\
6\end{array}$ & 岕 & & 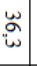 & & & 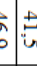 & w & 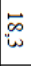 & 峲 & 岕 & & & 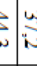 & & & & $\begin{array}{l}\vec{*} \\
+ \\
+\end{array}$ & El Salvador \\
\hline 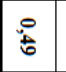 & 岕 & & & 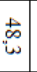 & & & 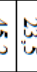 & ư & 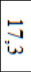 & $\begin{array}{l}\sim \\
\tilde{\omega} \\
-\infty\end{array}$ & 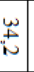 & & & 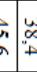 & & & & $\begin{array}{l}+ \\
\infty \\
\circ \\
\sigma\end{array}$ & Guatemala \\
\hline : & $\begin{array}{l}\tilde{N} \\
\text { 草 }\end{array}$ & $\overrightarrow{\mathrm{a}}$ & & $\begin{array}{c}\underset{\infty}{\omega} \\
\text { is }\end{array}$ & & t. & 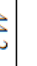 & & & & & 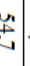 & 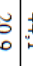 & 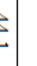 & & & & י & Haití \\
\hline$\stackrel{0}{\circ}$ & $\underset{\sim}{\tilde{\infty}}$ & & & 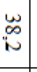 & & 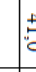 & 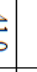 & $\underset{\sim}{w}$ & 0 & $\begin{array}{c}n \\
\cdots \\
\infty \\
\infty\end{array}$ & 岕 & & & 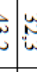 & & & & 品 & Honduras \\
\hline ఏ & $\begin{array}{c}\cong \\
-\infty \\
-\infty\end{array}$ & 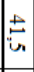 & 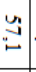 & 衤 & ". & $\vec{b}$ & $\vec{\infty}$ & يَّ & ك & : & $\underset{\infty}{+}$ & \pm & $E$ & 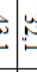 & & ta & & $\stackrel{\square}{*}$ & México \\
\hline : & $\begin{array}{l}\tilde{m} \\
0\end{array}$ & $\mid \begin{array}{c}\mathbf{w} \\
+ \\
\infty\end{array}$ & & : & & & 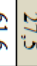 & 孛 & 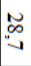 & 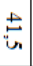 & : & & $t$ & $\$$ & & & & o & Nicaragua \\
\hline$\stackrel{\circ}{\circ}$ & $\begin{array}{l}\underset{\tilde{\sigma}}{0} \\
\end{array}$ & 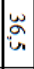 & & : & & $\breve{~}$ & 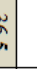 & $\stackrel{E}{E}$ & 2 & 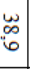 & ڤ & & & 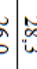 & & ic & & 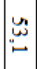 & Panamá \\
\hline 望 & $\underset{+\infty}{\tilde{N}}$ & & \begin{tabular}{|c|c|}
$\underset{s}{u}$ \\
is
\end{tabular} & & 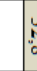 & ల్) & & & & & & & & & & 1 & & 离 & Paraguay \\
\hline 胥 & $\underset{N}{\mathbb{N}}$ & 状 & 空 & $\stackrel{0}{a}$ & 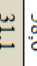 & 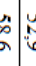 & s: & 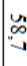 & 古 & t & "ू. & $\underset{\mathrm{C}}{\mathrm{t}}$ & & & & & & 离 & Perú \\
\hline$\stackrel{\circ}{6}$ & $\underset{\substack{\mathbb{\infty} \\
\infty \\
\infty}}{\mathbb{N}}$ & 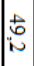 & & 岕 & & 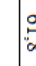 & 2 & 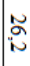 & " & & & & & y & & & & $\stackrel{2}{\omega}$ & R. Dominicana \\
\hline : & $\stackrel{\Xi}{\Xi}$ & $\$$ & 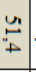 & 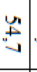 & 㐌 & 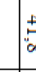 & 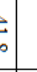 & $\begin{array}{lll}\infty & \\
0 & & \end{array}$ & & & & 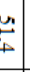 & & & & & & 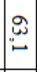 & Uruguay \\
\hline$\stackrel{\circ}{H}$ & \begin{tabular}{l}
$\tilde{\infty}$ \\
\hdashline \\
\hdashline
\end{tabular} & $\begin{array}{l}\text { U. } \\
\text { वे }\end{array}$ & : & 岁 & a. & 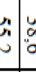 & & & & & & 㐘 & & & & & & $\infty$ & Venezuela \\
\hline & & $\begin{array}{c}\mathbb{w} \\
\infty \\
\infty\end{array}$ & $\mid \begin{array}{l}\vec{w} \\
6 \\
b \\
\end{array}$ & $\vec{b}$ & $\begin{array}{l}0 \\
6 \\
0\end{array}$ & 2 & 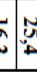 & & & & & & & & & & & & $?$ \\
\hline
\end{tabular}

Fuente: Elaboración personal en base a datos de los censos de población de los países de América Latina y del Proyecto IMILA del CELADE. 